\title{
A Survey of the Literature on Unintended Consequences Associated with Health Information Technology: 2014-2015
}

\author{
K. Zheng', J. Abraham², L. L. Novak ${ }^{3}$, T. L. Reynolds', A. Gettinger ${ }^{5}$ \\ 1 Department of Informatics, Donald Bren School of Information and Computer Sciences, University \\ of California, Irvine, Irvine, CA, USA \\ 2 Department of Biomedical and Health Information Sciences, College of Applied Health Sciences, \\ University of Illinois at Chicago, Chicago, IL, USA \\ 3 Department of Biomedical Informatics, School of Medicine, Vanderbilt University, Nashville, TN, USA \\ 4 School of Information, University of Michigan, Ann Arbor, MI, USA \\ 5 Office of the National Coordinator for Health Information Technology, U.S. Department of Health and \\ Human Services, Washington DC, USA
}

Results: The literature search identified 1,538 papers that were potentially relevant; 34 were deemed meeting our inclusion criteria after screening. Studies described in these 34 papers took place in a wide variety of care areas from emergency departments to ophthalmology clinics. Some papers reflected several previously unreported unintended consequences, such as staff attrition and patients' withholding of information due to privacy and security concerns. A majority of these studies (71\%) were quantitative investigations based on analysis of objectively recorded data. Several of them employed longitudinal or time series designs to distinguish between unintended consequences that had only transient impact, versus those that had persisting impact. Most of these unintended consequences resulted in adverse outcomes, even though instances of beneficial impact were also noted. While care areas covered were heterogeneous, over half of the studies were conducted at academic medical centers or teaching hospitals. Conclusion: Recent studies published in the past two years represent significant advancement of unintended consequences research by seeking to include more types of health IT applications and to quantify the impact using objectively recorded data and longitudinal or time series designs. However, more mixed-methods studies are needed to develop deeper insights into the observed unintended adverse outcomes, including their root causes and remedies. We also encourage future research to go beyond the paradigm of simply describing unintended consequences, and to develop and test solutions that can prevent or minimize their impact.

\section{Keywords}

Unintended consequences; health information technology; patient safety; electronic health records; medical order entry systems; Health Information Technology for Economic and Clinical Health Act

Yearb Med Inform 2016:13-29 http://dx.doi.org/10.15265/IY-2016-036 Published online November 10, 2016

\section{Introduction}

It has been extensively documented that introduction of health information technology (IT) is often associated with effects that are not intended by software designers, implementers, healthcare administrators, or clinicians; also known as "unintended consequences" or "e-iatrogenesis" [1-4]. While such effects can be beneficial, a majority of the health IT-related unintended consequences reported in the extant literature is found to cause adverse outcomes, such as new types of patient safety risks (e.g., wrong patient selection), as a result of poorly designed user interface, disrupted workflow, and communication breakdown $[5,6]$; and increased workload for clinicians (e.g., additional documentation demand), as a result of new regulatory requirements enabled and subsequently enforced by the use of health IT [7, 8].

Unintended adverse consequences became a particularly pronounced issue in mid-2000's with emerging evidence demonstrating that they directly or indirectly contributed to adverse patient safety events, near misses, and unsafe conditions [5, 6, 9-11]. In recent years, this concern has escalated as many industrialized countries began to implement policies and incentive programs to promote rapid adoption of health IT across all hospitals and clinics [2]. For example, two years after the commencement of the "Meaningful Use" program as part of the 2009 Health Information Technology for Economic and Clinical Health (HITECH) Act, the U.S. Office of the National Coordinator for Health Information Technology requested the Institute of Medicine (IOM) to convene a special committee to evaluate health IT-related safety concerns, resulting in a landmark report "Health IT and Patient Safety: Building Safer Systems for Better Care" [12]. Around the same time, the U.S. 
Agency for Healthcare Research and Quality contracted the RAND corporation to create a guide to help practitioners reduce unintended consequences when using electronic health records (EHR) [13].

As previous work has pointed out, "unintended" and "unanticipated" should not be treated as synonyms in this context [2]. Unintended consequences emphasize that a technological intervention exerts a temporary or enduring effect deviating from its original design intent [2]. For example, while one of the widely anticipated benefits of implementing EHRs is to improve work efficiency, their initial deployment often introduces a transitory, negative impact on productivity as users adapt to the technology. Even though this temporary loss of productivity is not intended (and unwanted), this "ramp-up" effect could and should be anticipated by any organization attempting to introduce a new technology in a new environment. However, the magnitude of this productivity loss may be observed at a level that is unacceptable and unanticipated by the implementation team, or continue on for a prolonged period of time with no clear sign of improvement. In such scenarios, unintended adverse consequences are not anticipated, warranting close attention and careful treatment. It should be noted that by this logic, unanticipated consequences are always unintended, as unanticipated outcomes are not obtainable by conscious design.

This review, conducted in early 2016, aims to summarize relevant research development in the past two years (2014-2015) to report on the trends of this steam of research, areas in which unintended consequences have continued to be reported, and common themes that emerge from the findings of these studies. Based on the conceptual framework above, we define the inclusion criteria as follows.

- First, the reported consequences must be unintentional, i.e., not planned by software designers or by the implementation team. These include unintended effects exerted on intended outcomes dimensions (e.g., a reminder system designed to improve the frequency of weighing pediatric patients was instead found to decrease the frequency). They also include effects exerted on unintended, perhaps seemingly unrelated outcomes dimensions (or "collateral effects," e.g., an electronic alert designed to reduce drug-drug interaction errors was found to cause an unexpected increase of other types of medication errors).

- Second, the intervention under investigation must be 'digital,' i.e., software applications (e.g., EHR) or hardware systems integrated with software systems (e.g., bar-code medication administration). By this definition, implementation of a managerial protocol on improving team coordination, or new clinical guidelines on mammographic screening, are considered out of the scope. When such managerial protocols and clinical guidelines were indeed implemented through a digital form (e.g., as an electronic checklists or as computerized reminders), we use our best judgment to determine if the evaluation focused on the stimuli itself, or on the delivering methods (i.e., health IT).

- Third, the impact of the intervention must be assessed using valid quantitative, qualitative, or mixed-methods designs; and the unintended consequences observed, if quantifiable, must be substantial. For example, if a computerized prescriber order entry (CPOE) system implemented to improve medication safety was found to cause no or statistically insignificant change, then it is not deemed within the scope of this review. However, if the study also included a rigorous qualitative investigation that led to the discovery of unintended consequences accounting for the ineffectiveness of the intervention, then it may be considered within the scope.

- Fourth, while relevant position papers, commentaries, and policy briefs are cited throughout this paper, a study to be included in the review must be original empirical investigation of an intervention that had been deployed in the field for routine or for trial use. By this definition, this review excludes opinions and thought pieces that may have offered only anecdotal evidence, and unintended effects reported in laboratory testing of early technology prototypes that did not involve naturalistic settings and real users.
However, survey studies seeking general perceptions (e.g., of the potential privacy and security risks that may be associated with health IT) are included, even if some respondents may not have direct experience with the technology studied.

This paper is organized as follows. The next section, Background, presents a brief overview of the history of relevant research on health IT-related unintended consequences, in addition to existing literature surveys and systematic reviews. Then, the Methods section describes the approach we used in searching literature databases to retrieve relevant papers, and the review process. The Results section summarizes each paper reviewed, and the key findings organized into relevant thematic groups. Finally, in the Discussion section, the authors offer their reflections on new research development pertinent to the topic in 2014 and 2015, as well as gaps and recommendation for future directions.

\section{Background}

\section{A History of Relevant Research}

While use of computers in healthcare can be traced back much earlier, health informatics research as a scientific domain emerged around the 1960 's, producing a proliferation of medical diagnostic systems based on early breakthroughs in artificial intelligence [14, 15]. However, due to the lack of supporting infrastructures at the time, such as hospital information systems that capture and store patient data crucial to diagnostic accuracy and relevance, most of these early-generation diagnostic systems did not make their way into everyday clinical practice [14].

In late 1990's, a second wave of health IT research and practice arrived [16]. This new wave was marked by several milestone papers published in prestigious medical journals that unequivocally demonstrated the practical value of more modern forms of health IT such as computerized order entry and decision support [17]. For example, through a randomized controlled trial, Bates et al. (1998) showed that a CPOE system 
deployed at the Brigham and Women's Hospital (Boston, MA, USA) decreased the rate of non-intercepted serious medication errors by more than half [18]. In another study, also conducted at Brigham and Women's, Teich et al. (2000) found that $\mathrm{CPOE}$ coupled with computerized decision support was effective in altering physicians' prescribing behavior, resulting in improvements along multiple dimensions such as guideline adherence and overdose prevention; and these improvements persisted at one- and two-year follow-up [19]. The demonstrated early successes of these modern health IT systems inspired many other healthcare organizations to pursue similar technologies. In the U.S., reports from the Institute of Medicine raised awareness of quality gaps and the harms inflicted on patients by the healthcare system, fueling a movement to support error-prone human practitioners with computational support for tasks and decisions [20,21]. This movement was further stimulated by an executive order signed by then Bush Administration calling for most Americans to have electronic health records by 2014 [22]; and by a 2005 RAND report that predicted significant cost-savings if health IT were adopted nationwide $[23,24]$. At the global stage, the World Health Assembly passed a Resolution (WHA 58.28) in 2005, acknowledging that eHealth (i.e., information and communication technologies) is a cost-effective tool for improving patient care delivery and public health. The Resolution urged its member states to consider developing and implementing eHealth services in the various areas of the health sector [25].

Despite early warnings about cultural and behavioral barriers, lack of user friendliness, and potential errors due to automated decision support/making [26-28], many healthcare organizations rushed to acquire health IT in order to become fully 'wired' and 'paperless' [29]. During this time, vendor-supplied commercial systems became prevalent, as few provider institutions had the capacity to develop homegrown systems customized to their needs and constraints. However, implementing off-the-shelf IT products, at large scales, in very complex healthcare organizations, was confronted with fierce challenges that many did not anticipate. It was during this period that unintended adverse consequences associated with health IT began to surface.

Drawing upon a literature review and a series of qualitative studies conducted in the U.S., The Netherlands, and Australia, Ash et al. (2004) began to systematically document instances in which health IT systems might foster errors, rather than reduce their likelihood [1]. They found that health IT-induced errors are common in two processes: the process of entering and retrieving information and the process of communication and coordination. A year later, Koppel et al. (2005) studied another deployment of CPOE and found that use of the system could facilitate 22 new types of medication safety risks, ranging from information errors - caused by data fragmentation and failure to integrate the CPOE with other hospital IT systems; in addition to human-machine interface flaws - originating in machine rules that do not correspond to work organization or usual behavior [5]. In the same year, Han et al. (2005) unveiled an unexpectedly increased mortality rate in a children's hospital that appeared to coincide with the implementation of a commercial CPOE system; speculated reasons for it included chaotic workflow post-implementation, e.g., "hard-stop" alerts that inhibited proper actions from being taken during emergency situations; and reduced opportunities of communication among clinicians, especially between physicians and nurses [6]. Despite controversies in statistical inference and causality analysis [30], this paper received an enthusiastic response from the informatics community praising the authors' courage to report their findings and calling for deeper investigation into the underlying sociotechnical root causes of the observed unintended consequences [31].

These early research accounts spurred many follow-up studies that led to a surge of publications produced on the topic in a relatively short period of time [9-11,32-36], putting the issue in a national spotlight. The discussions culminated in 2009 when the American Medical Informatics Association (AMIA) decided to devote its Annual Health Policy Meeting to unintended consequences of health IT. A consensus report out of the meeting was published in the subsequent year [2]. The report recognized the severity and the magnitude of the issue. It also expressed a salient concern that most knowledge known about unintended consequences at the time was from a small number of early adopters. With the accelerated uptake of health IT at the national level on the horizon because of the 2009 HITECH ACT, there was an urgent need for additional research on unintended consequences of health IT and a concerted effect to collect, refine, and disseminate research findings to practitioners.

\section{B Relevant Literature Surveys and Systematic Reviews}

While there has been a significant body of work published on unintended consequences associated with health IT, literature reviews, systematic reviews, and meta-analyses dedicated to the topic have been surprisingly scarce. Besides the 2009 IOM report and the 2010 AMIA Health Policy Meeting report that included an in-depth review of the relevant literature $[2,12]$, we were only able to locate a few related reviews.

Pirnejad, Bal, and Shahsavar (2010) surveyed the scholarly work published before September 2009 and identified 26 relevant papers [37]. The unintended consequences reported in these papers were classified into four general areas: workflow, communication, technical, and user-related. Carling et al. (2010) conducted another review in the same year, focusing on problematic prescriptions and adverse outcomes that may be associated with electronic medication management systems used in ambulatory care [38]. By reviewing 38 studies that met the inclusion criteria (18 of them were randomized controlled trials), the authors concluded that the scientific evidence available at the time was not adequate to substantiate fears of additional patient safety risks as a result of using IT; however, the authors also concluded it was premature to reject such a hypothesis, due to the lack of evidence. In the following year, Harrington et al. (2011) searched the literature published between 2000 and 2009 and identified 24 relevant studies [29]. The authors concluded in the review that hospitals at the time were under tremendous pressure to implement health IT because of its "demonstrated and presumed 
improvements to patient safety." However, unintended adverse consequences, especially those that presented a threat to patient safety, were evident based on the authors' analysis of the literature, and should be considered.

Three other reviews were published more recently. In Voshall et al. (2013), the authors examined nurses' behavior working around barcode medication administration systems [39]. They found 13 studies describing this behavior, concluding that workarounds were common in nursing practice, which might negate the benefits of barcode medication administration and facilitate new types of errors. Another review by Gephart, Carrington, and Finley (2015) focused on nurses' negative experience with use of EHRs [40]. Only five studies were identified, however. These studies reported several adverse effects affecting nursing work such as undesirable workflow, constantly changing requirements for work due to imperfect EHR design, and difficulties in accessing necessary information at the point of decision-making. The last review, by Marcilly et al. (2015), looked into how usability flows of medication alerting systems may link to usage problems and consequent unintended adverse outcomes [41]. Based on a peruse of 26 relevant publications, the authors found that usability flows (e.g., low signal-to-noise ratio of medication alerts) are responsible for a wide range of usage issues (e.g., increased workload and information involuntarily missed), which in turn results in unintended consequences that have detrimental effects on workflow, technology effectiveness, the medication management process, and patient safety.

Despite a shortage of review articles on unintended consequences, it is worth noting that since 2003, a large number of systematic reviews have become available to summarize the effort of implementing and evaluating health IT across different time periods and different types of applications (e.g., EHR, CPOE, health information exchange) [42-105]. With only a few exceptions [102-105], the results of the great majority of these reviews suggested dismissal of a once widely-held belief that use of health IT would lead to significant gains in efficiency, quality of care, patient safety, and cost containment. Even though the potential was acknowledged, most of these studies concluded that the demonstrated effectiveness of health IT systems, as how they were implemented, "is not compelling and is limited by modest study sample sizes and designs" [54]. Some studies also noted that most early successes of health IT were reported by a handful of benchmark institutions of internally developed systems, and the benefits might have been selectively reported [43, 80]; casting a serious doubt on how well the results could be replicated among average healthcare organizations.

In response to the criticism that the benefits of health IT yielded in the past decade have been modest at best, a group of RAND researchers published a paper to offer their opinions why the company's 2005 projection fell short [106]. The authors speculated that this disappointing performance could be attributed to several factors: shortcomings in the design and implementation of health IT systems, the reluctance of clinicians to invest the time and effort required to master difficult-to-use technology, and the failure of healthcare providers and institutions to reengineer care processes to reap the full benefits of health IT [106].

\section{Methods and Materials}

To identify relevant studies, we searched three literature databases: MEDLINE, Embase, and CINAHL. A paper to be included in the review must be in English, and must be published, either in print or as an electronic publication ahead of print, between January 1, 2014 and December 31, 2015.

The query used a set of keywords to ensure that the intervention(s) under investigation is pertinent to health IT, e.g., "health [information technology, IT]," "electronic [health, medical] records," "computerized [physician, provider, prescriber] order entry," "[computerized clinical] decision support," "e-prescribing," as well as their anonyms and spelling variants. In the first round of search we included keywords such as "unintended consequences" and "unintended outcomes." These keywords however proved to be too limiting, as the query failed to retrieve several relevant papers published in the target timeframe that we are aware of. A look into these papers revealed that the authors did not explicitly label their study objective, or research findings, as "unintended consequences" of health IT. Therefore, we revised our search query to use more general terms, such as "evaluation" and "implementation," in order to capture a broader range of health IT implementation and evaluation studies.

The results of the two rounds of literature search were then consolidated for subsequent screening. Each paper was independently reviewed by at least two authors, first by title and abstract and then by full text. The most challenging part of the screening was to determine if the results reported in a study met the definition of "unintended consequences of health IT" (see the Introduction section). We used our best judgment to decide if the outcomes observed were truly unintentional; if the impact was substantial; if the intervention was 'digital;' and if the study was an original empirical investigation conducted based on an intervention deployed and used in the field. Equivocal cases were discussed until consensus was reached; marginally relevant papers were always kept rather than dismissed.

\section{Results}

The two rounds of search resulted in a total of 1,535 distinct titles ( 755 from 2014, and 780 from 2015).

Out of the 1,535 papers screened, 1,444 papers were deemed not meeting the inclusion criteria. Over half of the papers excluded are studies about secondary use of electronic patient care data; many on developing and evaluating new predictive models, [e.g., 107] information retrieval tools, [e.g., 108] or natural language processing algorithms [e.g., 109]. Among the remainder, many were not relevant to health IT, e.g., a study evaluating an intervention program to reduce medication preparation errors [110]; or health IT was not the main intervention or only served as a delivering platform for the intervention, e.g., a study evaluating technical assistance and financial incentives alongside EHR implementation [111]. When health IT was indeed the fo- 
cus of the study, the results were either in line with the researchers' projection (i.e., intended) or were not statistically significant [e.g., 112]; or health IT was tested in simulated laboratory environments instead of being evaluated in situ in the field [e.g., 113]. This left us 34 papers to include in this review [8, 114-146]. Figure 1 shows the PRISMA diagram exhibiting the literature search and screening processes.

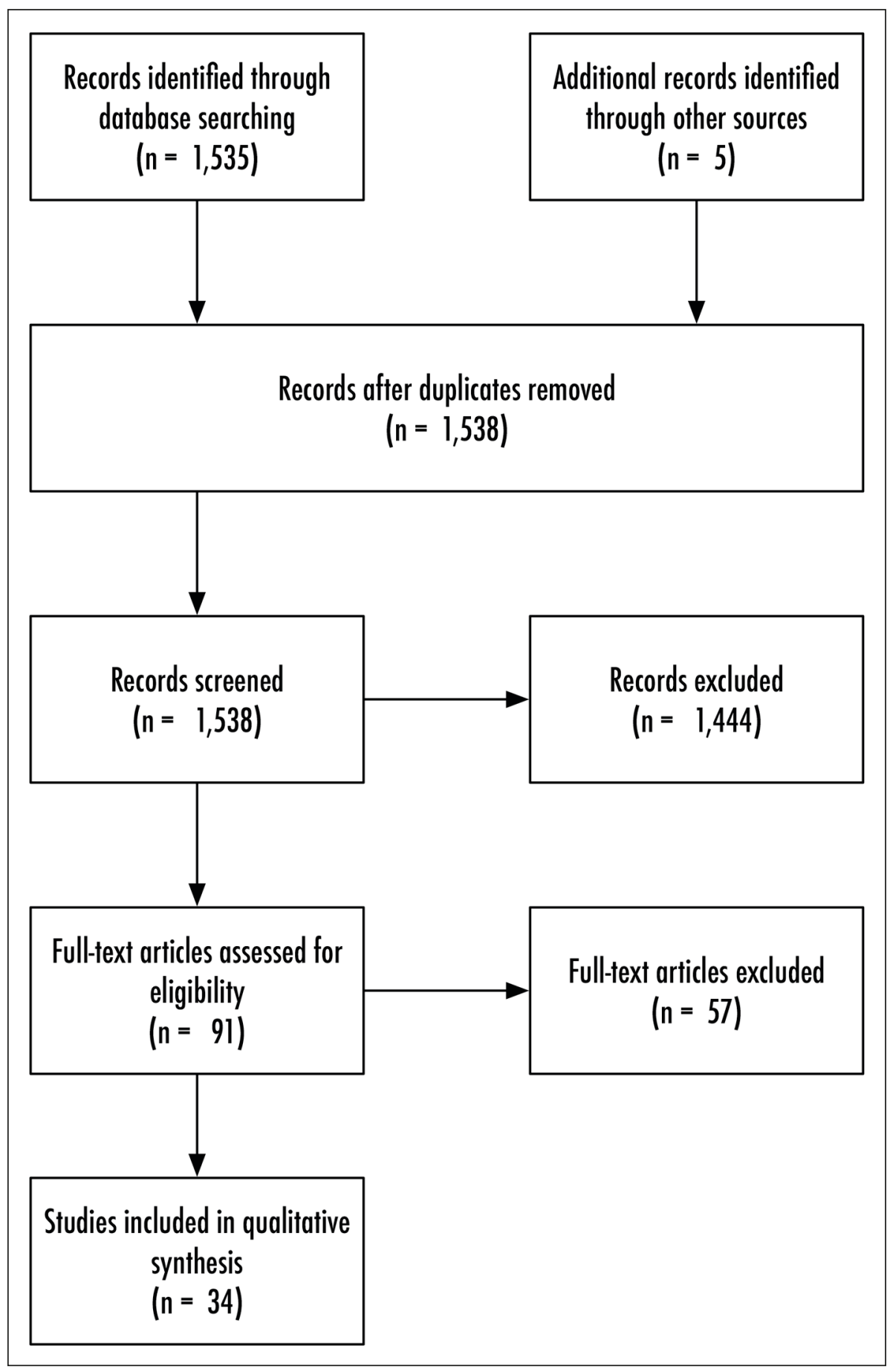

Fig. 1 Literature search and screening processes

\section{A Overall Trends}

Table 1 lists the 34 papers. Nine studies were conducted outside of the U.S. [116, 118, 121, 127, 130, 137-139, 140]. Care settings included were rather heterogeneous, but ambulatory care and emergency department (ED) were studied relatively more often. Compared to early work that predominantly focused on $\mathrm{CPOE}$, more studies included in our review evaluated the impact of EHR systems (23 out of 34, or $68 \%$ ). This change may not necessarily suggest a shifted focus of research, though. It may be simply because many healthcare organizations have completed the transition from standalone CPOE systems to more comprehensive EHRs, of which ordering and order management functions have become an integral part.

Unlike early research that was primarily qualitative, a majority of these recent studies attempted to quantify the unintended impact associated with health IT implementation or use: out of the 34 studies, 24 (71\%) were quantitative investigations; 2 employed mixed methods. Electronic charts and time and motion observations were the most common sources of quantitative data; and pre-post comparison was the predominant design. No studies that we reviewed were based on randomized controlled trials.

Below, we summarize each of the papers reviewed according to the nature of the unintended consequences identified. Note that these study summaries are brief, therefore may not cover all respects of the discussions contained in the paper (e.g., outcomes that demonstrated no change before and after health IT implementation may not be included in the summary). Also note that while we organized the papers under different subsections, representing different areas of impact, some unintended consequences are interrelated and may affect multiple outcomes dimensions. For example, unintended changes in workflow or in team coordination may have significant implications on patient safety and quality of care.

\section{B Patient Safety}

Whether health IT implementation is linked with escalated patient safety risks continues to be a focal point of recent research. Eight out of the 34 papers $(23 \%)$ were dedicated to this topic, especially on health IT's adverse effects on medication safety. 


\begin{tabular}{|c|c|c|c|c|c|c|c|c|c|c|c|}
\hline 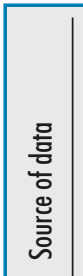 & 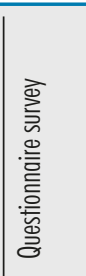 & 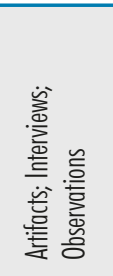 & 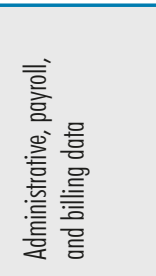 & $\begin{array}{l}\text { 믐 } \\
\text { 咳 }\end{array}$ & 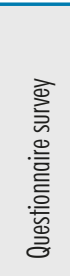 & 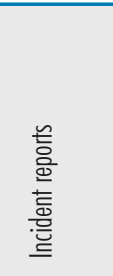 & 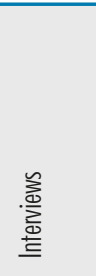 & 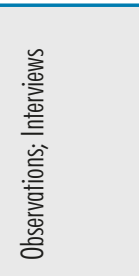 & $\begin{array}{l}\text { 흘 } \\
\text { 咅 } \\
\text { 흘 } \\
\text { 흔 }\end{array}$ & & 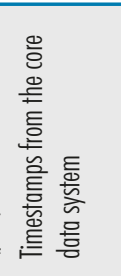 \\
\hline 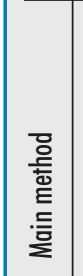 & 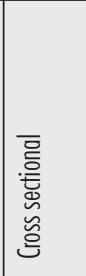 & 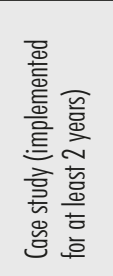 & 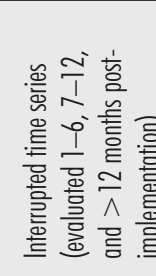 & 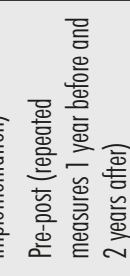 & $\begin{array}{l}\overline{\underline{c}} \\
\text { 产 } \\
\text { 总 }\end{array}$ & 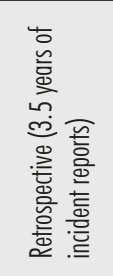 & 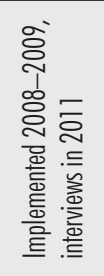 & 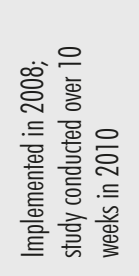 & 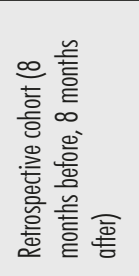 & 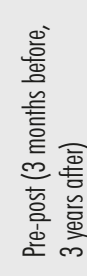 & 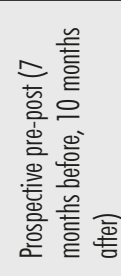 \\
\hline $\begin{array}{l}\text { 离 } \\
\text { 离 } \\
\text { 言 }\end{array}$ & 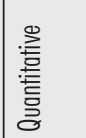 & 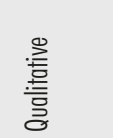 & $\begin{array}{l}\text { 䇏 } \\
\text { 言 }\end{array}$ & $\begin{array}{l}\text { 諅 } \\
\text { 言 }\end{array}$ & 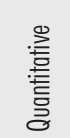 & 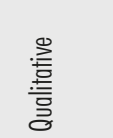 & 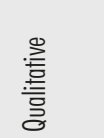 & $\begin{array}{l}\text { 善 } \\
\text { 喜 }\end{array}$ & $\begin{array}{l}\text { 堷 } \\
\text { 言 }\end{array}$ & 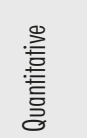 & 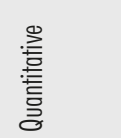 \\
\hline
\end{tabular}
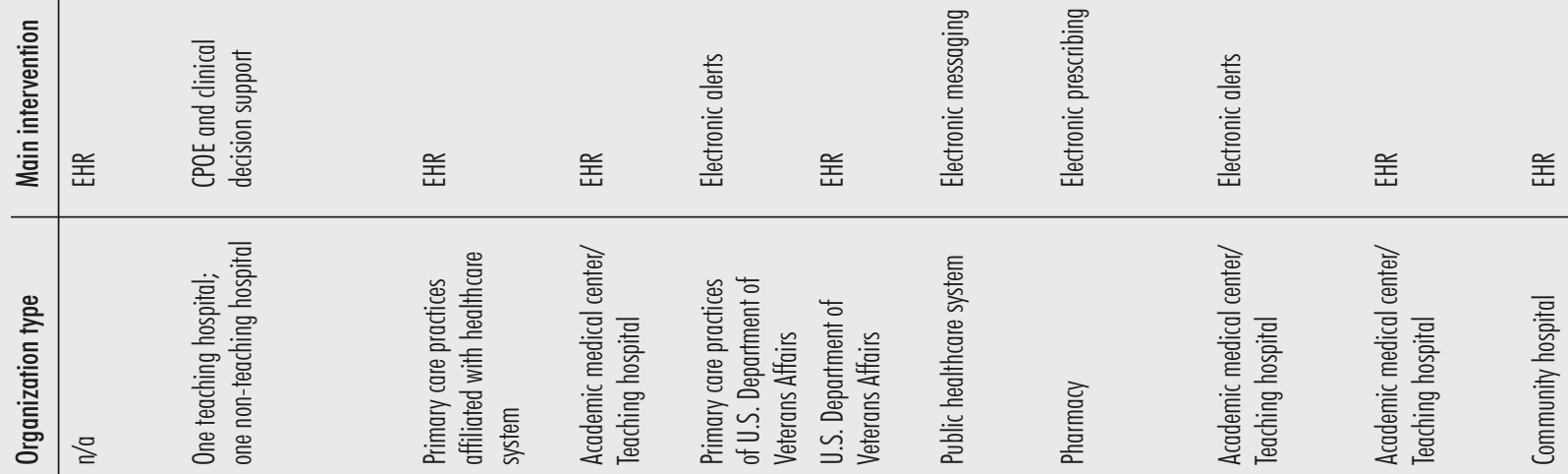

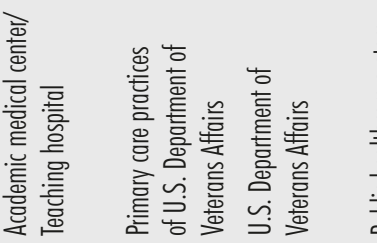
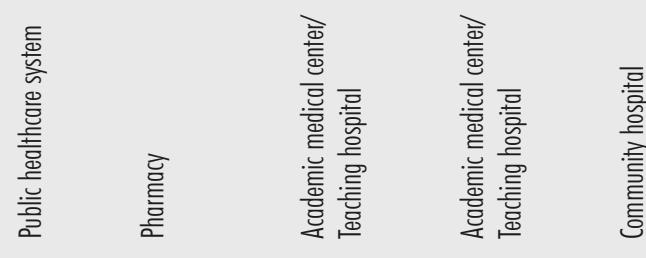

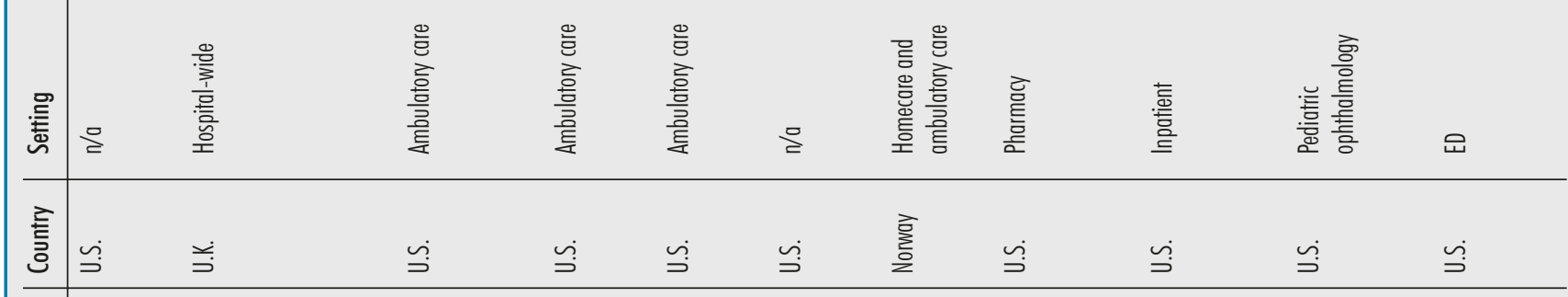

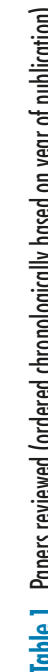

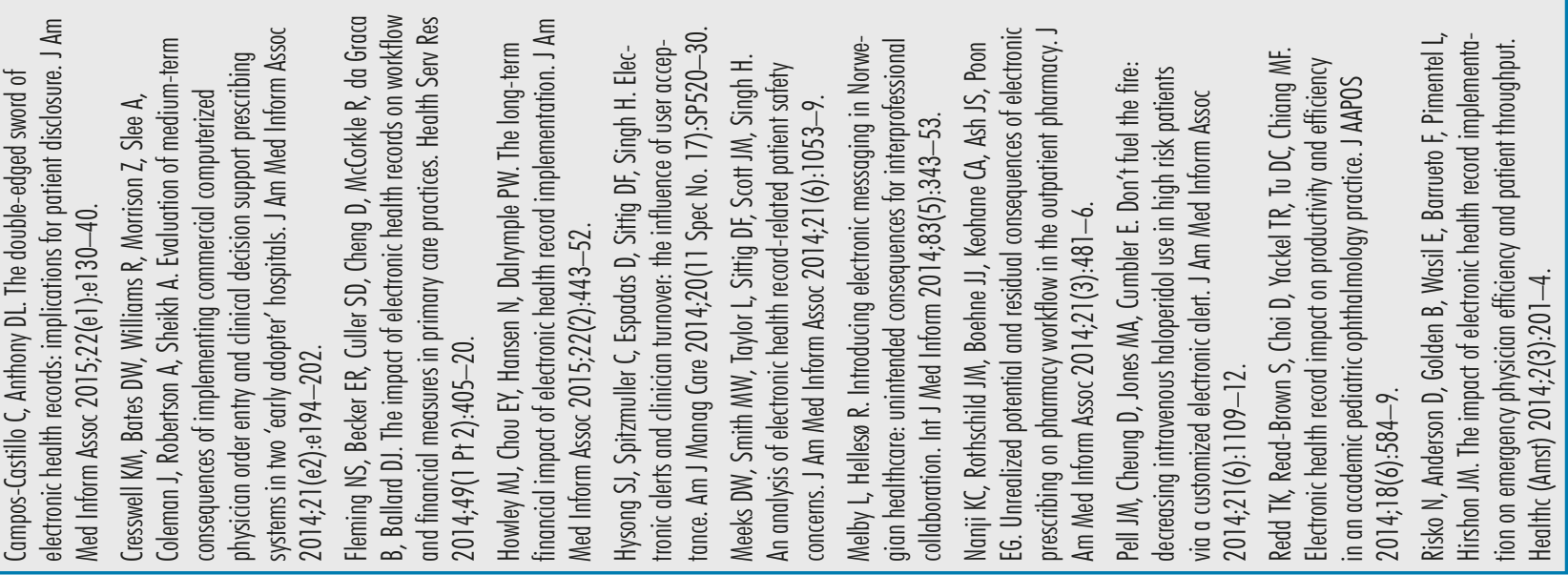




\begin{tabular}{|c|c|c|c|c|c|c|c|c|c|c|c|}
\hline 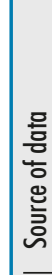 & 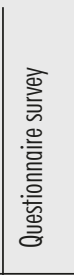 & 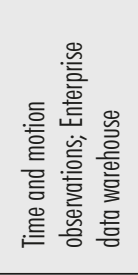 & $\begin{array}{l}\text { 誉 } \\
\text { 薏 }\end{array}$ & 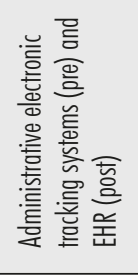 & 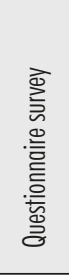 & 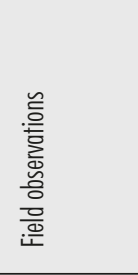 & 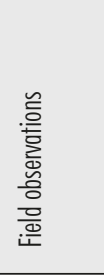 & 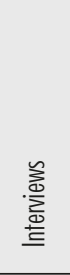 & 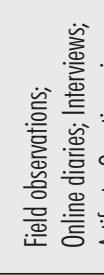 & 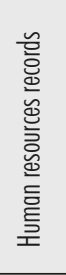 & 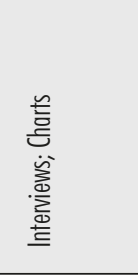 \\
\hline 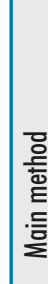 & 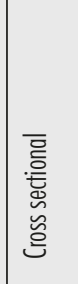 & 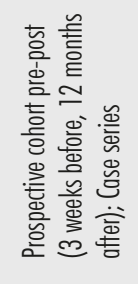 & 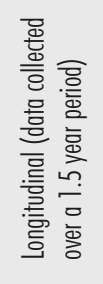 & 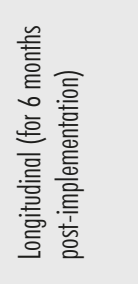 & 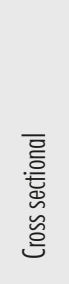 & 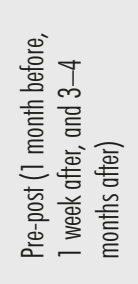 & 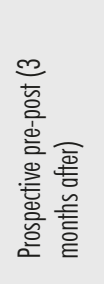 & $\stackrel{\unrhd}{\Xi}$ & 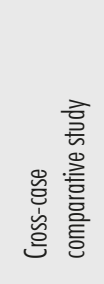 & 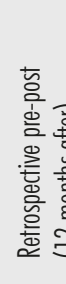 & 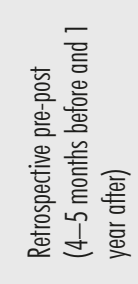 \\
\hline 曾 & 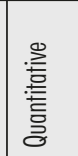 & 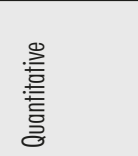 & 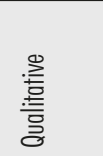 & 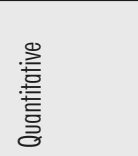 & $\begin{array}{l}\text { 㝘 } \\
\text { 言 }\end{array}$ & 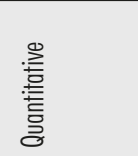 & $\begin{array}{l}\text { 諳 } \\
\text { 言 }\end{array}$ & 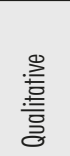 & 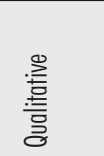 & 言 & 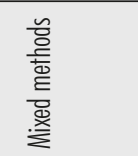 \\
\hline
\end{tabular}
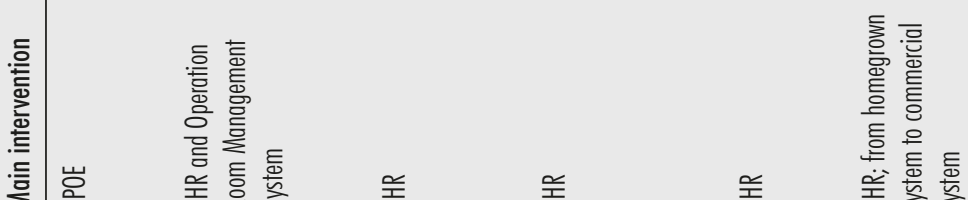

\begin{tabular}{|c|c|c|c|c|c|c|c|c|c|c|}
\hline 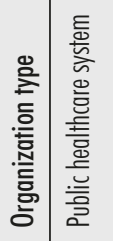 & 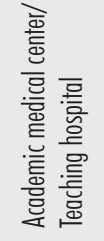 & 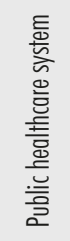 & 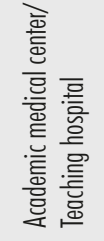 & $\stackrel{\unrhd}{\stackrel{2}{2}}$ & 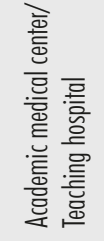 & 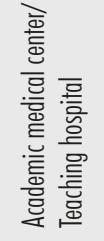 & $\begin{array}{l}\text { 㖖 } \\
\text { 章 }\end{array}$ & 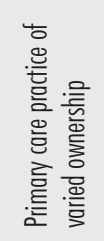 & 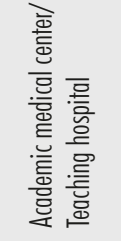 & 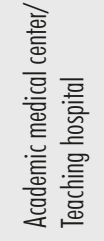 \\
\hline
\end{tabular}

\begin{tabular}{|c|c|c|c|c|c|c|c|c|c|c|c|}
\hline 䓂 & \begin{tabular}{|l} 
离 \\
产 \\
言 \\
豪
\end{tabular} & 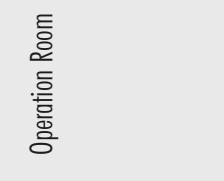 & 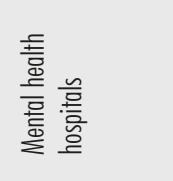 & $\mathrm{C}$ & $\stackrel{g}{\Xi}$ & 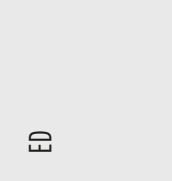 & $\underline{\underline{z}}$ & $\stackrel{g}{g}$ & 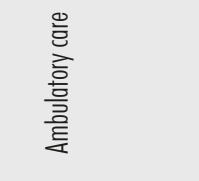 & $\stackrel{\Xi}{\Xi}$ & 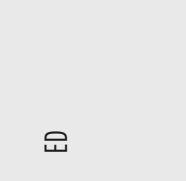 \\
\hline 言 & 㖣婄 & $\stackrel{山}{s}$ & 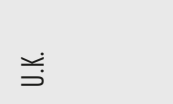 & $\circlearrowleft$ & $\stackrel{\leftrightarrow}{\Xi}$ & $\stackrel{\cup}{\Xi}$ & $\stackrel{\cup}{=}$ & $\stackrel{\Delta}{=}$ & $\stackrel{\Delta}{=}$ & $\stackrel{\sim}{=}$ & 毫 \\
\hline & 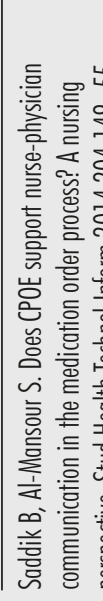 & 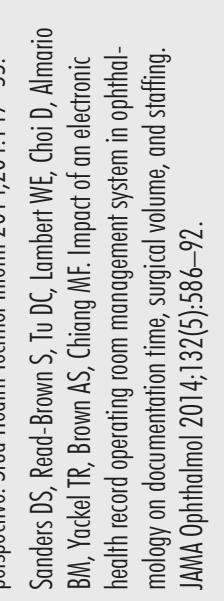 & 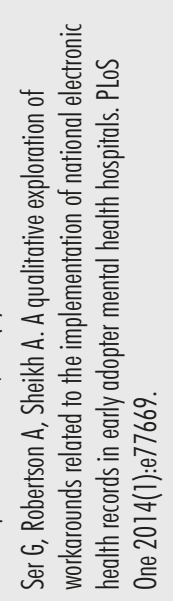 & 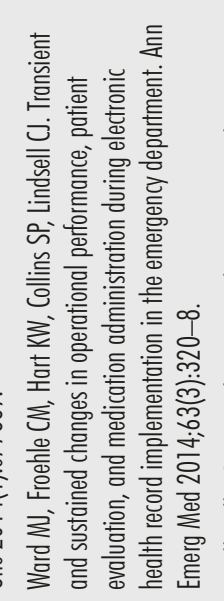 & 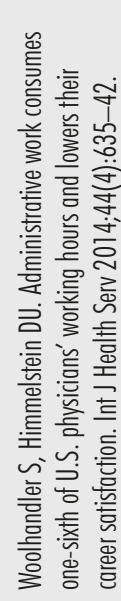 & 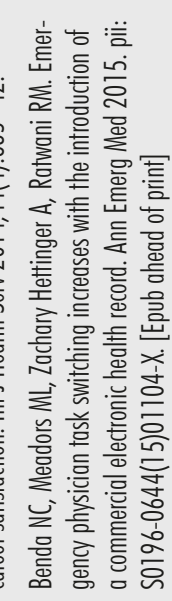 & 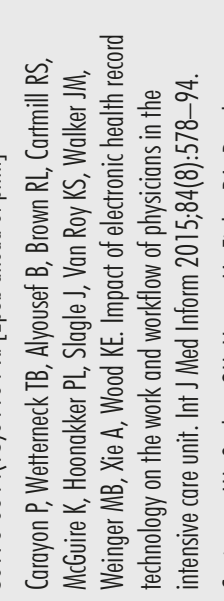 & 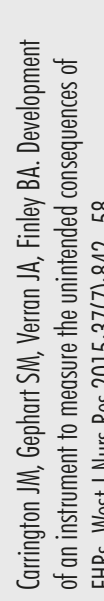 & 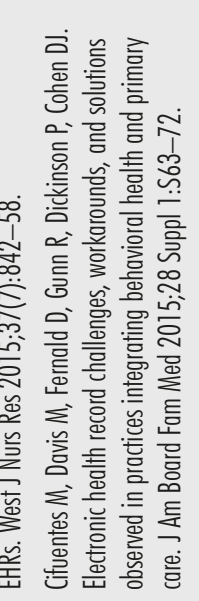 & 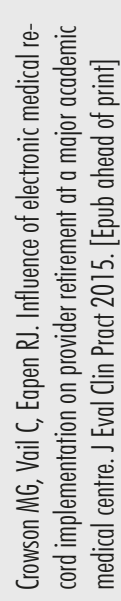 & 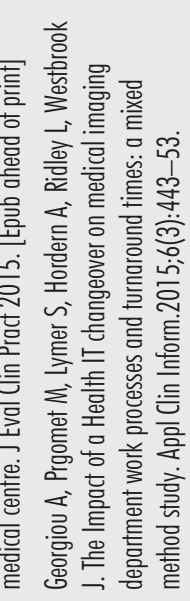 \\
\hline
\end{tabular}




\begin{tabular}{|c|c|c|c|c|c|c|c|c|c|c|c|c|}
\hline 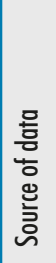 & 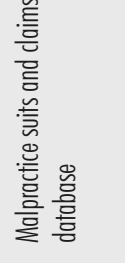 & 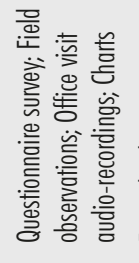 & & 旁 & 竧 & 毫 & 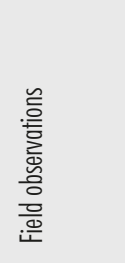 & 咅 & 善 & 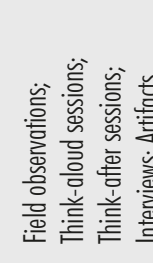 & 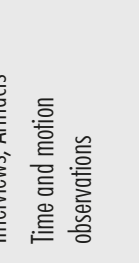 & 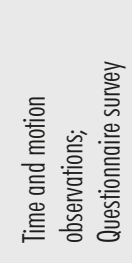 \\
\hline 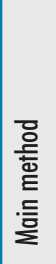 & 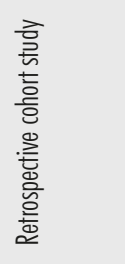 & $\stackrel{\square}{g}$ & 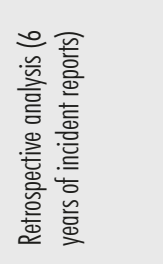 & 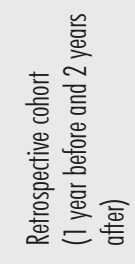 & 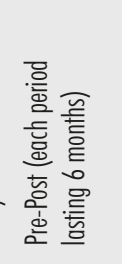 & 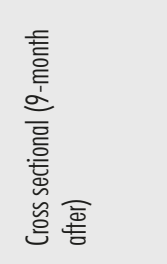 & 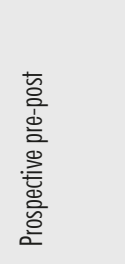 & 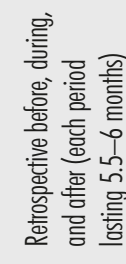 & 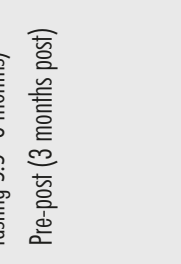 & 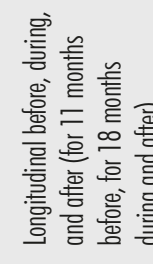 & 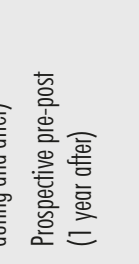 & $\stackrel{\Xi}{\rightleftarrows}$ \\
\hline 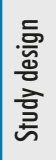 & 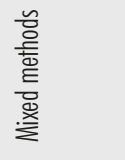 & $\begin{array}{l}\text { 总 } \\
\text { 喑 } \\
\text { 言 }\end{array}$ & 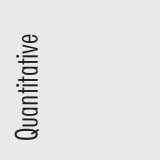 & $\begin{array}{l}\text { 总 } \\
\text { 謍 } \\
\text { o }\end{array}$ & $\begin{array}{l}\text { 諅 } \\
\text { 言 }\end{array}$ & $\begin{array}{l}\text { 总 } \\
\text { 言 } \\
\text { 言 }\end{array}$ & $\begin{array}{l}\text { 誊 } \\
\text { 言 } \\
\text { 言 }\end{array}$ & $\begin{array}{l}\text { 总 } \\
\text { 譬 } \\
\text { o }\end{array}$ & $\begin{array}{l}\text { 誊 } \\
\text { 衰 } \\
\text { 言 }\end{array}$ & 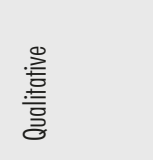 & 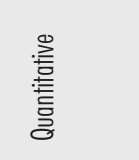 & $\begin{array}{l}\text { 总 } \\
\text { 䚻 } \\
\text { 言 }\end{array}$ \\
\hline 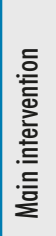 & 姜 & 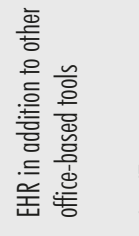 & 氡 & 姜 & 岁 & 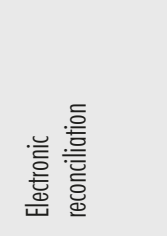 & 뭉 & 姜 & 姜 & 姜 & 姜 & 姜 \\
\hline 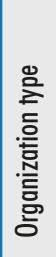 & $\stackrel{\varrho}{!}$ & 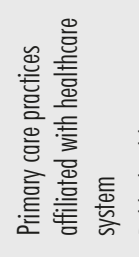 & 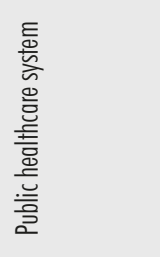 & 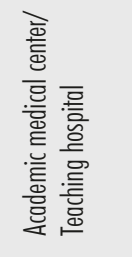 & 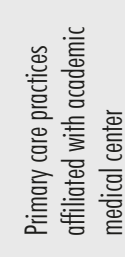 & 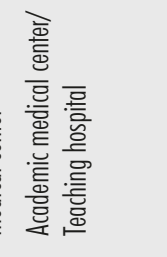 & 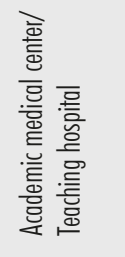 & 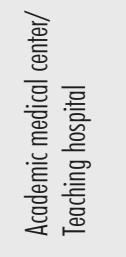 & 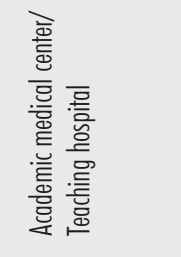 & 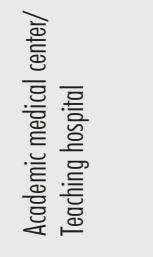 & 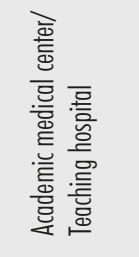 & 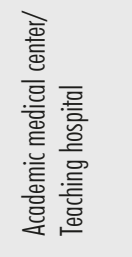 \\
\hline 胥 & $\stackrel{\Xi}{!}$ & $\begin{array}{l}\text { 产 } \\
\text { 言 } \\
\text { 言 } \\
\text { 言 }\end{array}$ & $\stackrel{\Xi}{\rightleftarrows}$ & 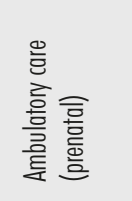 & $\begin{array}{l}\text { Oू } \\
\text { 言 } \\
\text { 言 } \\
\text { 言 }\end{array}$ & $\begin{array}{l}\text { 言 } \\
\text { 言 } \\
\text { 晋 }\end{array}$ & 름 & 을 & $\begin{array}{l}\text { 言 } \\
\text { 竟 } \\
\text { 亘 }\end{array}$ & 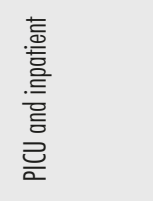 & 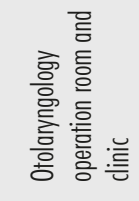 & $\begin{array}{l}\text { 产 } \\
\text { 䇏 } \\
\text { 至 }\end{array}$ \\
\hline 衰 & $\circlearrowleft$ & $\stackrel{\cup}{S}$ & 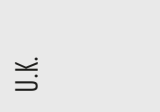 & $\stackrel{\cup}{S}$ & $\cong$ & $\begin{array}{l}\text { 믈 } \\
\text { 喜 } \\
\text { 要 }\end{array}$ & $\stackrel{\cup}{S}$ & $\cong$ & $\cong$ & 믈 & $\stackrel{\sim}{S}$ & 믈 \\
\hline & 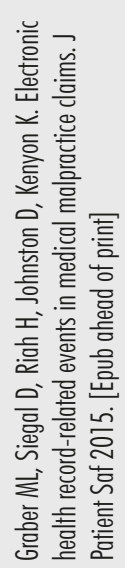 & 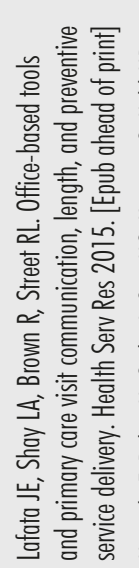 & 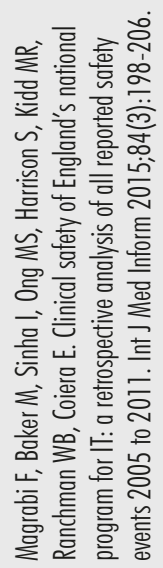 & 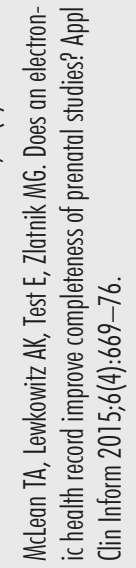 & 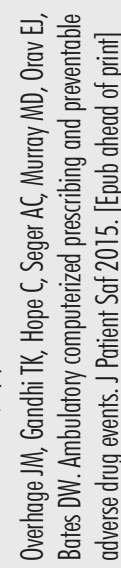 & 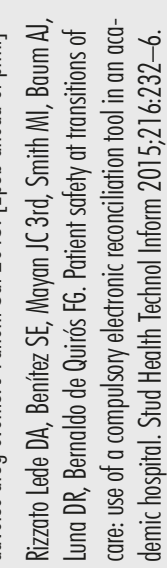 & 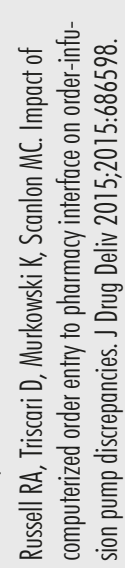 & 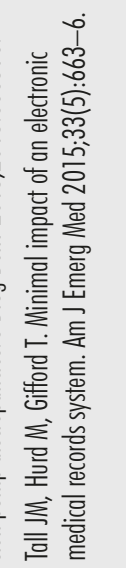 & 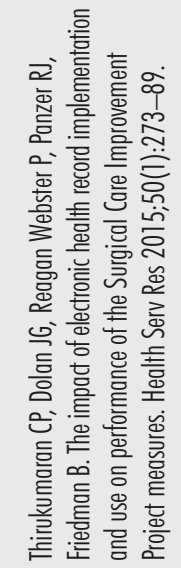 & 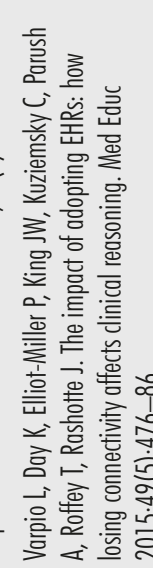 & 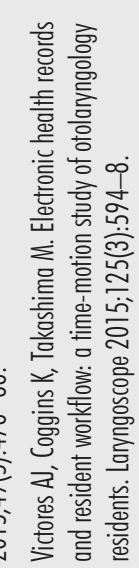 & 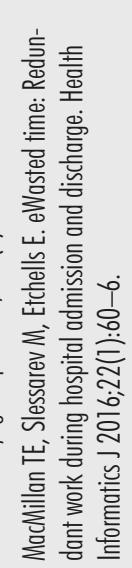 \\
\hline
\end{tabular}


Among the eight papers there were four quantitative studies. Pell et al. (2014) evaluated the effectiveness of introducing a computerized alert customized to reduce adverse drug events (ADE) among hospitalized patients with prolonged QTc [114]. While the alert was found to be effective in correcting inappropriate orders for intravenous haloperidol, it also caused occasional inappropriate discontinuation of the medication among certain patients (e.g., patients receiving end-of-life care), for whom its use was justified. Russell et al. (2015) examined potential discrepancies between intravenous fluid (IVF) orders and bedside infusion pump settings in a pediatric intensive care unit (PICU) [115]. The results show that fewer discrepancies were present after implementing a new, bidirectional interface between the CPOE system used in the PICU and the pharmacy information system. However, an unexpected increase was observed in the rate of omitted medications as well as wrong dosing. Rizzato et al. (2015) studied an electronic medication reconciliation tool deployed at an academic medical center in Argentina [116]. The authors found that while the use of the tool contributed to better quality of medication lists, it also led to accidental removal of active medications, which could impose severe threats to patient safety. In another study, Overhage et al. (2015) compared the rates of ADEs before and after implementing a computerized prescribing system across 17 ambulatory primary care practices at two study sites. The results show that the use of the system was associated with a $56 \%$ reduction in the potential $\mathrm{ADE}$ rate at one study site; however, at the other site, it was associated with a $104 \%$ increase of potential ADEs [117].

Two qualitative studies conducted during this period sought to investigate the nature and root causes of new patient safety risks associated with health IT. Through document analysis, interviews, and observations, Cresswell et al. (2014) studied the process of implementing CPOE and medication-related decision support at two hospitals in the U.K. [118]. Their analyses unveiled a range of IT-induced patient safety risks including unsafe workarounds; additional security measures at odds with the contingent and highly pressured work routines; confusing and inflexible user interface designs; duplicate prescribing; and reduction in team-wide discussions. In another study, Nanji et al. (2014) qualitatively assessed the impact of an electronic prescribing system implemented in an outpatient pharmacy [119]. By analyzing the collected observation notes and interview transcripts, the authors identified 26 unintended consequences that they categorized under five themes: communication, workflow disruption, cost, technology, and opportunity for new errors.

Three studies published in this period used incident reports and malpractice suits and claims as a source of research data to identify adverse events attributable to health IT vulnerability. By analyzing a large malpractice database, Graber et al. (2015) found 248 cases of malpractice suits and claims filed in 2012 and 2013 that were linked with health IT use, most of which were related to medication errors, misdiagnoses, and treatment complications [120]. The authors also reported four common sources of origin of health IT vulnerabilities: the danger inherent in hybrid systems and EHR conversions; the danger of delayed, missing, or incorrect data, services, or actions; the danger of over-reliance on the EHR; and the inherent risks using copy/ paste functionality, overriding alerts, and employing workarounds. Another similar study by Magrabi et al. (2015) scrutinized the 2005-2011 incident reports received by an IT safety team in the U.K., about half of which were originally submitted to the U.K. national IT help desk [121]. The authors found that $68 \%$ of the reports described potentially hazardous circumstances. A majority of them were associated with technical rather than human factors. The third study, by Meeks et al. (2015), analyzed incidents tracked by a non-punitive, voluntary reporting system used at the U.S. Veterans Health Administration [122]. The authors found that about two-thirds of the incidents were linked with unsafe technology; the remaining due to unsafe use of technology. Most of the safety concerns described in the paper were attributable to problematic display of EHR data, software upgrades or modifications, data transmission between different components of the EHR, and 'hidden dependencies' within the system.

\section{Time Efficiency and Workflow}

"More/new work for clinicians" and "unfavorable workflow issues" are two most common unintended consequences of health IT reported in early qualitative research [7]. Several studies published in 2014 and 2015 attempted to quantify their effects, using methods such as retrospective chart reviews and time and motion observations.

Four such studies were conducted in the ED setting. Two of them used a longitudinal design that involved data collection at multiple time points after introducing the intervention. Both found that EHR adoption was associated with short-term negative effects, even though these effects diminished over time. The first study, by Risko et al. (2014), examined how EHR implementation affected the rapid assessment and management of life threatening conditions in the ED at two hospitals [123]. The authors found that the median patient processing time increased immediately after the implementation, and then slowly returned to and eventually dropped below the pre-implementation levels. The second study, by Ward et al. (2014), also found a transient disruption caused by EHR implementation, reflected as increased length of stay; decreased patient satisfaction; and increased rates of medication administration, laboratory testing, and overall radiologic imaging [124]. While the length of stay and patient satisfaction measures returned to the baseline after 4 to 8 weeks, the increased rates of medication administration and the utilization of advanced testing persisted.

The third ED study, by Benda et al. (2015), conducted time and motion observations in an ED before, during, and after it transitioned from a homegrown EHR to a vendor-supplied commercial system [125]. The results show that the new system did not incur a redistribution of physicians' time to different tasks (e.g., from direct care to indirect care), but the number of tasks that these physicians engaged in per minute increased substantially. The authors argued that higher rates of task switching could increase the cognitive burden on physicians, which might result in adverse consequences such as stress and mistakes. The fourth study conducted in the ED, by 
Tall, Hurd, and Gifford (2015), analyzed monthly census reports to assess how ED's work efficiency was affected before, during, and after the implementation of an enterprise-wide EHR system at a community hospital [126]. The authors found a modest decrease in the total ED length of stay for patients who were admitted to the hospital, and an increased time in the $E D$ for transfer patients. The percentage of patients who left ED without being seen also increased significantly.

Several other studies took place in nonED environments. In their paper entitled "eWasted time: Redundant work during hospital admission and discharge," MacMillan, Slessarev, and Etchells (2016) described a time and motion study conducted at an academic medical center in Canada [127]. The aim was to quantify EHR-related redundant work during admission and discharge to a general internal medicine service. As the title of the paper implies, a significant proportion of clinician time was found to be unnecessarily "wasted" on tasks such as duplicative data entry due to EHR use (note that even though this paper appeared in print in 2016, it has been available as an electronic publication ahead of print since 2014). Redd et al. (2014) studied the impact of EHR implementation in a pediatric ophthalmology clinic, and found that the implementation had a negative impact on productivity and efficiency, such as an $11 \%$ drop of overall clinical volume [128]. The authors also found that nearly half of the charts were closed outside normal business hours (30\% on weekdays, $14 \%$ on weekends). Similarly, Sanders et al. (2014) evaluated the impact of an EHR and an Operation Room Management system [129], and found intraoperative nursing documentation time significantly increased especially in shorter procedures. Georgiou et al. (2015) used a mixed-methods design to assess the impact of deploying a picture archiving and communication system and a radiology information system [130]. The study, conducted in a medical imaging department in Australia, shows that turnaround time was significantly reduced, representing efficiency gains. However, assimilation of new systems with existing work processes was considered inadequate and in some instances unsafe. For example, images became instantaneously available to physicians anywhere at the hospital while the radiologist's report was still being prepared. This raised concerns of potential misinterpretation of the images, which could lead to incorrect diagnoses.

Among the studies conducted in non-ED settings, two used a time and motion design. Carayon et al. (2015) observed residents and attending physicians' work before and after implementing a comprehensive EHR with built-in order management and physician documentation functionalities in an ICU [131]. The results show that EHR use had significant impact on ICU physician work and workflow. After adopting the EHR, both residents and attending physicians spent more time on clinical review and documentation. EHR implementation also caused an increased rate of task switching for residents, and changed temporal flow of tasks. In the other study, Victores, Coggins, and Takashima (2015) conducted time and motion observations with otolaryngology residents to assess the impact of EHR on their workflow [132]. The authors found that the overall resident efficiency was not significantly altered. However, more time post-implementation was shifted from directly caring for patients to documenting in the EHR during clinic days (as opposed to operative days).

The last study addressing the work efficiency issue used a different approach. By surveying a nationally representative sample of 4,720 U.S. physicians, Woolhandler and Himmelstein (2014) assessed how much time physicians devote to administrative tasks, how it relates to their career satisfaction, and the role that adoption of EHR plays [8]. The results of the survey suggest that more extensive use of EHR was indeed associated with a higher level of administrative burden, which in turn led to lower career satisfaction by physicians. The authors concluded that the federal mandate of EHR adoption in the U.S., in combination with other policy changes such as a shift to employment in large practices and the increasing prevalence of financial risk sharing, would likely increase physicians' paperwork burdens and thus contribute to their career dissatisfaction.

\section{Documentation Quality, Clinician Performance, and Quality of Care}

Several studies focused on quality-oriented measures such as documentation quality, clinician performance, and quality of care. Through qualitative observations across eight primary care clinics and three community mental health centers, Cifuentes et al. (2015) found that current EHR systems were inadequate to support integrated behavioral health and primary care, due to their insufficient ability to document and track all relevant behavioral and physical health information; facilitate communication and coordination; and exchange information with other devices and other EHRs [133]. To accommodate these limitations, clinicians employed numerous workarounds such as double data entry, which could be linked with unintended efficiency loss and increased chances for errors. In another study, Lafata et al. (2015) quantitatively assessed the impact of EHR use on ambulatory primary care by analyzing 485 office visits with 64 primary care physicians [134]. The results show that patients seen by providers with an EHR had longer visits and received fewer guideline-recommended preventive care services. While the authors did not investigate reasons underlying this quantitative finding, they suggested that "screen-driven" information gathering (i.e., the provider's dialog with the patient is driven by the order of the screens and information that the computer displays) [147] might be a contributing factor, as an EHR-driven dialog could lead to missed opportunities for comprehensive inquiry and risk factor assessments.

In McLean et al. (2015), the authors described a chart-review study that compared documentation rates of prenatal HIV and purified protein derivative (PPD) tests before and after an EHR system was deployed [135]. The results show that in the year following the EHR implementation, there was a significant drop in the documentation rate of PPD tests. While the situation improved in the second year, the rate did not compare more favorably to that of paper charts used at baseline. No substantive change was observed for HIV tests. Similarly, when studying the impact 
of EHR implementation at a tertiary-care teaching hospital, Thirukumaran et al. (2015) also found a significant temporary reduction in surgical quality immediately following the EHR implementation, even though all quality indicators returned to their baseline levels three months after the implementation [136].

Lastly, Varpio et al. (2015) conducted a qualitative study to examine how patient data presented in an inpatient EHR system might affect clinical reasoning [137]. The authors found that while the EHR was effective in collecting dispersed data, it failed to display the data back to clinicians in a cognitively efficient matter that would facilitate clinical reasoning. In particular, they found that the electronic flowsheet provided in the EHR overly emphasized individual data values, diminishing the information linking the data chronologically and with other relevant data elements. By contrast, old paper flowsheets emphasized chronology and data interconnectedness, which are critical to efficient and informed decision-making.

\section{E Communication and Coordination}

Several studies presented in the earlier sections touched upon health IT's unintended consequences on communication and coordination, with most findings being negative [118, 119, 133]. However, Melby and Hellesø (2014) reported predominantly positive unintended effects after introducing an e-message tool at a Norwegian hospital [138]. By interviewing clinicians, clerks, and managers, the authors found that the tool encouraged clinicians and staff to become more proactive in communicating with others. They also found that after implementing the tool, nurses perceived more weight to their requests as e-messages were automatically archived as part of patient records. Similarly, Saddik and Al-Mansour (2014) reported mixed experiences by nurses from a hospital in Saudi Arabia where $\mathrm{CPOE}$ functions were implemented as part of a comprehensive EHR system [139]. Many nurses participating in the study agreed that the newly implemented CPOE functions supported their workflow and enhanced nurse-physician communication; however, they also needed to follow up with physicians more frequently on medication orders after the implementation. Additionally, the study found that nurses with longer years of experience, and those from the surgery department, did not perceive the benefits of using computerized ordering.

\section{F Workarounds}

Workarounds are common tactics employed by end users to circumvent limitations imposed by health IT [11]. Certain workarounds can facilitate clinical work by mitigating the influence of poorly designed software systems. However, they may also engender practices that are unsafe $[11,39]$. Among the studies that we reviewed, several described clinicians' working-around behavior in response to various hindering conditions created by adoption of technology [118, 120, 133]. Further, the study by Ser, Robertson, and Sheikh (2014) was dedicated to seeking examples and explanations of health IT-induced workarounds [140]. Through interviewing stakeholders involved in implementing or using an EHR system at two early adopter mental health hospitals in the U.K., the authors found that workarounds, such as deferred data entry and deliberately entering data in wrong places, were common. They also found that a wide range of operational, cultural, organizational, and technical factors contributed to clinicians' decision to work around an IT application rather than using it as prescribed.

\section{G Financial Impact}

Two studies assessed the potential unintended financial impact of adopting health IT. Fleming et al. (2014) analyzed the administrative, payroll, and billing data collected from 26 primary care practices across multiple time points before and after the implementation of an ambulatory EHR [141]. The results show that staffing and practice expenses increased following the implementation, although after excluding software maintenance cost the magnitude of the increase appeared to be small. Further, while productivity, patient volume, and net income decreased initially, they all recovered to the baseline levels after 12 months. The authors concluded that the longer-time productivity and financial performance of the study practices were not affected by the EHR implementation; however, the short-term negative impact was evident. In another study, Howley et al. (2015) analyzed the productivity and reimbursement data collected longitudinally from 30 ambulatory care practices for 2 years after implementing an EHR system [142]. The authors found that, by comparing each site to their preEHR baseline, the study practices saw fewer patients with the EHR, but their reimbursements significantly increased.

\section{H Staff Attrition}

End user dissatisfaction (and related symptoms such as negative emotions, stress, and anxiety) was discovered very early on as one of the unintended adverse consequences that may be associated with health IT adoption $[9,10]$. Previous survey studies also indicated that this dissatisfaction could lead to personnel attrition issues including staff turnover or early retirement. For example, in a 2011 survey conducted in the U.S., 12\% of the pediatric urologists responding to the survey expressed that they would retire early if EHR use became a federal mandate [148]. In this review, two studies were specifically conducted to address the question whether providers and other types of healthcare workers might choose to leave the workforce due to difficulties in adapting to health IT.

Through surveying primary care providers employed by the U.S. Department of Veterans Affairs, Hysong et al. (2014) assessed providers' perception of EHR-based alert notifications, and how this perception might be linked with their intentions to quit and turnover [143]. The authors found that monitoring/feedback, i.e. "degree to which employee's performance using EHR-based alerting systems is monitored and to which feedback is provided," is a strong predictor of intention to quit. Further, they found that the perceived value of EHR-based alert notifications also predicts intention to quit, indirectly through its effects on provider satisfaction; and is directly linked 
with voluntary turnover. In another study, Crowson, Vail, and Eapen (2015) investigated staff attrition at a large academic medical center [144]. The analysis was based on three-year monthly provider attrition data collected before and after implementing a commercial EHR that replaced an in-house developed system. The results show that a peak in provider departure occurred in the month immediately before the EHR was implemented. Median age of the providers who left during this period was higher than that of the providers who departed at other times.

\section{Privacy and Confidentiality}

One study included in our review assessed whether EHR adoption may adversely affect patients' willingness to disclose information due to privacy and security concerns. By surveying a nationally representative patient sample in the U.S., Campos-Castillo and Anthony (2015) found that 13\% of the respondents reported having withheld information from a provider to protect against the perceived EHR privacy and security risks [145]. After accounting for the respondents' global ratings of care, the authors confirmed a positive relationship between patients withholding information and their physician using an EHR during the patient encounter.

\section{J Methods Development}

Only one study was devoted to methodological development. Carrington et al. (2015) described their effort developing a survey questionnaire to quantify unintended consequences that may be experienced by nurses [146]. The instrument contains constructs identified from the authors' prior empirical work (which is why we deemed this paper to be with the scope of this review). These constructs include perceived barriers (e.g., hardware issues, data entry, and irretrievability) and nurse-initiated solutions (e.g., documentation shortcuts and saving without signature). Psychometric testing of the instrument was not reported in the paper, but was said to be currently underway.

\section{Discussion}

Research in the past decade has unequivocally demonstrated the existence of unintended consequences that may accompany health IT's implementation and use $[2,12]$. The studies published in the recent two years contribute more evidence. These studies report a wide range of unintended effects introduced by many different types of health IT applications across many different types of care areas and organizational settings. With a few exceptions [e.g., 138], most of the unintended consequences reported in these studies were associated with adverse outcomes.

The recent research included in this review differs from early work in several distinctive ways. First, more studies sought to quantify the impact of health IT using objectively recorded data such as patient census, billing claims, and human factors records. Quantitative results from these studies, although constrained by the quality and direct applicability of data sources, have greatly enhanced the existing knowledge base mostly contributed by early studies based on qualitative accounts. Qualitative accounts are an indispensable means for discovering unintended consequences of health IT and the mechanisms by which they can produce harm. However, they often fall short of measuring the magnitude of their impact, and can be more susceptible to prejudices (e.g., reluctance to change) and biases (e.g., cognitive heuristics and recall errors). Second, compared to early work, the scope of research had expanded considerably in the past two years. The studies that we reviewed evaluated several new types of health IT applications, such as e-messaging and electronic medication reconciliation; covered more care areas, such as ophthalmology clinics and mental health hospitals; and examined additional dimensions of unintended outcomes, such as financial impact, staff attrition, and patients' withholding of information due to privacy and security concerns. Collectively, these studies enriched the body of research on unintended consequences and extended beyond its traditional focus on CPOE and medication safety. Third, leveraging the increasing availability of clinical environments that had been exposed to health IT for longer periods of time, several studies involved longitudinal or time series designs to collect repeated measurements at multiple time points post-implementation $[124,125,129,135,136,141,142]$. These repeated measurements enabled researchers to distinguish temporary effects during and immediately following health IT implementation, from longer-term impact after the technology had been fully assimilated into clinical practice and end users' job routines. This knowledge would help researchers and practitioners better understand the life span of different types of unintended consequences so that more effective remedy strategies can be developed accordingly.

Our analysis of the recent literature also revealed several methodological limitations yet to be addressed. More than half of the studies were conducted at academic medical centers or teaching hospitals. These settings have very unique characteristics (e.g., resident rotations) that are not commonly found in other types of healthcare organizations. Generalizability of the results obtained from studying these settings may be therefore limited. Further, while the quantitative studies contributed richer knowledge to the existing evidence base, very few studies used a mixed-methods design to explain what was observed quantitatively. As a result, questions such as what accounted for the unintended consequences, and how to mitigate their adverse impact, could only be answered with anecdotes or authors' speculation; or were left unanswered in some of these studies. Additional mixed-methods research is therefore needed, to not only quantify the impact of unintended consequences, but also develop a better understanding of their root causes and measures that can be used to counter their adverse effects.

To this end, we encourage future research to go beyond the paradigm of proving the existence of unintended consequences, and to start developing and testing solutions that can prevent or minimize their impact [e.g., 149]. We believe that, based on the evolution of this body of research in the past decade, a widely held consensus has been reached that unintended adverse consequences brought by health IT implementation are almost inevitable, even if their effects may be temporary and the magnitude of the impact may be 
small $[2,12,150]$. Therefore, any healthcare organizations preparing for new health IT uptake should always anticipate such effects and have plans in place accordingly [151]. In addition, all stakeholders need to work together to continue to improve and enforce the standardization of terminologies and information exchange protocols. Vendors should also invest more in improving the usability of their software, and reducing the barrier to sharing data within their own systems and with other systems.

Even though gaps remain [152], we are now seeing efforts initiated toward these directions. The U.S. Office of the National Coordinator for Health Information Technology, for example, has funded a series of studies examining the safety aspects of health IT and suggesting strategies to safely implement health IT [150, 153-158]. Several papers cited in this review, including the IOM report on health IT and patient safety [12], are a direct result of this investment. Other federal agencies in the U.S., such as the National Institute of Standards and Technology, the Agency for Healthcare Research and Quality, and the Food and Drug Administration, joined the effort and issued several guidelines to improve the usability of EHR systems $[159,160]$ and to monitor and curb their unintended adverse effects $[13,161]$. Another recent report, prepared by the National Quality Forum under a contract with the U.S. Department of Health and Human Services, provides guidance on identification and prioritization of health IT patient safety measures [162]. A coalition of disparate stakeholders has also come together to produce a roadmap to a Health IT Safety Collaborative [163]. Similar initiatives are being taken in the private sector as well. These range from "Partnership for Health IT Safety" spearheaded by the ECRI Institute [164]; to a recently announced million-dollar HeroX challenge to produce a safe, secure, and $100 \%$ accurate national patient identifier created by the College of Healthcare Information Management Executives [165].

This review has several limitations. First, as mentioned earlier, identifying published work reporting unintended consequences of health IT proved to be a very difficult task. In the title or the abstract, studies may not be explicit about their findings related to unintended consequences, as it may not be the main intended goal of the study. Further, studies may report relevant findings using an alternative language, such as "adverse impact on patient safety," which makes our keywords-based literature search difficult to conduct. Thus, despite best effort, this review may not be inclusive of all papers that have reported relevant results. Second, the scope of the review is restrained to a two-year window. This is a relatively short time period which may not be indicative of all recent development of the field. Third, to facilitate literature search and data analysis, we adopted a rather restrictive definition of unintended consequences. It is possible that our review left out some studies that do not meet the inclusion criteria yet present value to understanding the phenomenon of unintended consequences associated with implementation and use of health IT. Lastly, because this review only targeted original empirical investigations published in the scientific literature, we did not include thought pieces [e.g., 166], positions papers [e.g., 167], framework development [e.g., 168], books [e.g., 169], and policy briefs and government reports [e.g., 154-157, $160,161]$. These publications however may contain valuable information and insights. We therefore encourage readers to seek them out for a more comprehensive picture of the recent development in unintended consequences research.

\section{Conclusion}

We surveyed the 2014-2015 scientific literature to examine studies investigating unintended consequences associated with health IT implementation and use. We found that the research published in this period represents a significant leap forward by covering more care areas, more IT applications, and more dimensions of unintended outcomes. However, while more quantitative studies were conducted, few incorporated a qualitative component to gain deeper insights into what was quantitatively observed. More research using a mixed-methods design is therefore needed. We also encourage future research to deploy and evaluate solutions that prevent or minimize the impact of unintended consequences, rather than simply seeking more evidence to prove their existence.

\section{References}

1. Ash JS, Berg M, Coiera E. Some unintended consequences of information technology in health care: the nature of patient care information system-related errors. J Am Med Inform Assoc 2004;11(2):104-12.

2. Bloomrosen M, Starren J, Lorenzi NM, Ash JS, Patel VL, Shortliffe EH. Anticipating and addressing the unintended consequences of health IT and policy: a report from the AMIA 2009 Health Policy Meeting. J Am Med Inform Assoc 2011;18(1):82-90.

3. Palmieri PA, Peterson LT, Ford EW. Technological iatrogenesis: new risks force heightened management awareness. J Healthc Risk Manag 2007;27(4):19-24.

4. Weiner JP, Kfuri T, Chan K, Fowles JB. "e-iatrogenesis": the most critical unintended consequence of CPOE and other HIT. J Am Med Inform Assoc 2007;14(3):387-8; discussion 389.

5. Koppel R, Metlay JP, Cohen A, Abaluck B, Localio AR, Kimmel SE, et al. Role of computerized physician order entry systems in facilitating medication errors. JAMA 2005;293(10):1197-203.

6. Han YY, Carcillo JA, Venkataraman ST, Clark RS, Watson RS, Nguyen TC, et al. Unexpected increased mortality after implementation of a commercially sold computerized physician order entry system. Pediatrics 2005;116(6):1506-12.

7. Poissant L, Pereira J, Tamblyn R, Kawasumi Y. The impact of electronic health records on time efficiency of physicians and nurses: a systematic review. J Am Med Inform Assoc 2005;12(5):505-16.

8. Woolhandler S, Himmelstein DU. Administrative work consumes one-sixth of U.S. physicians' working hours and lowers their career satisfaction. Int J Health Serv 2014;44(4):635-42.

9. Campbell EM, Sittig DF, Ash JS, Guappone KP, Dykstra RH. Types of unintended consequences related to computerized provider order entry. J Am Med Inform Assoc 2006;13(5):547-56.

10. Ash JS, Sittig DF, Poon EG, Guappone K, Campbell E, Dykstra RH. The extent and importance of unintended consequences related to computerized provider order entry. J Am Med Inform Assoc 2007;14(4):415-23.

11. Koppel R, Wetterneck T, Telles JL, Karsh BT. Workarounds to barcode medication administration systems: their occurrences, causes, and threats to patient safety. J Am Med Inform Assoc 2008;15(4):408-23.

12. IOM (Institute of Medicine). Health IT and Patient Safety: Building Safer Systems for Better Care. Washington, DC: The National Academies Press; 2012.

13. Jones SS, Koppel R, Ridgely MS, Palen TE, Wu S, Harrison MI. Guide to Reducing Unintended Consequences of Electronic Health Records. (Prepared by RAND Corporation under Contract 
No. HHSA290200600017I, Task Order \#5). AHRQ Publication No. 11-0105-EF. Rockville, MD: Agency for Healthcare Research and Quality; 2011.

14. Engle RL Jr. Attempts to use computers as diagnostic aids in medical decision making: A thirty-year experience. Perspect Biol Med 1992;35(2):207-19.

15. Miller RA. Medical diagnostic decision support systems - past, present, and future: A threaded bibliography and brief commentary. J Am Med Inform Assoc 1994;1(1):8-27.

16. Berner ES, Detmer DE, Simborg D. Will the wave finally break? A brief view of the adoption of electronic medical records in the United States. J Am Med Inform Assoc. 2005;12(1):3-7.

17. Bates DW, Gawande AA. Improving safety with information technology. N Engl J Med 2003:348(25):2526-34.

18. Bates DW, Leape LL, Cullen DJ, Laird N, Petersen LA, Teich JM, et al. Effect of computerized physician order entry and a team intervention on prevention of serious medication errors. JAMA 1998(15):1311-6.

19. Teich JM, Merchia PR, Schmiz JL, Kuperman GJ, Spurr CD, Bates DW. Effects of computerized physician order entry on prescribing practices. Arch Intern Med 2000;160(18):2741-7.

20. Kohn LT, Corrigan JM, Donaldson MS, editors; Committee on Quality of Health Care in America; Institute of Medicine. To Err Is Human: Building a Safer Health System. Washington, DC: The National Academies Press; 2000

21. IOM (Institute of Medicine). Crossing the Quality Chasm: A New Health System for the 21st Century. Washington, DC: The National Academies Press; 2011.

22. Executive Order: Incentives for the Use of Health Information Technology and Establishing the Position of the National Health Information Technology Coordinator. http://georgewbush-whitehouse. archives.gov/news/releases/2004/04/20040427-4. html; accessed January 24, 2016.

23. Girosi F, Meili R, Scoville R. Extrapolating Evidence of Health Information Technology Savings and Costs. The RAND Corporation; 2005.

24. Hillestad R, Bigelow J, Bower A, Girosi F, Meili $\mathrm{R}$, Scoville R, et al. Can electronic medical record systems transform health care? Potential health benefits, savings, and costs. Health Aff (Millwood) 2005;24(5):1103-17.

25. World Health Assembly. Resolutions and Decisions: WHA58.28 eHealth. http://www.who.int/ healthacademy/media/WHA58-28-en.pdf?ua=1; accessed January 25, 2016.

26. Massaro, TA. Introducing physician order entry at a major academic medical center: I. Impact on organizational culture and behavior. Acad Med 1993;68(1):20-5.

27. Montgomery AA, Fahey T, Peters TJ, MacIntosh C, Sharp DJ. Evaluation of computer based clinical decision support system and risk chart for management of hypertension in primary care: randomised controlled trial. BMJ 2000;320(7236):686-90.

28. Goldstein MK, Hoffman BB, Coleman RW, Tu SW, Shankar RD, O'Connor M, et al. Patient safety in guideline-based decision support for hypertension management: ATHENA DSS. J Am Med Inform Assoc 2002; 9(6 Suppl 1):s11-s16.
29. Harrington L, Kennerly D, Johnson C. Safety issues related to the electronic medical record (EMR): synthesis of the literature from the last decade, 2000-2009. J Healthc Manag 2011;56(1):31-43; discussion 43-4.

30. Longhurst C, Sharek P, Hahn J, Sullivan J, Classen D. Perceived increase in mortality after process and policy changes implemented with computerized physician order entry. Pediatrics 2006;117(4):1450-1.

31. Sittig DF, Ash JS, Zhang J, Osheroff JA, Shabot MM. Lessons from "Unexpected increased mortality after implementation of a commercially sold computerized physician order entry system". Pediatrics 2006;118(2):797-801.

32. Ash JS, Sittig DF, Dykstra RH, Guappone K, Carpenter JD, Seshadri V. Categorizing the unintended sociotechnical consequences of computerized provider order entry. Int J Med Inform 2007;76 Suppl 1:S21-7.

33. Harrison MI, Koppel R, Bar-Lev S. Unintended consequences of information technologies in health care - an interactive sociotechnical analysis. J Am Med Inform Assoc 2007;14(5):542-9.

34. Sittig DF, Ash JS, Guappone KP, Campbell EM, Dykstra RH. Assessing the anticipated consequences of Computer-based Provider Order Entry at three community hospitals using an open-ended, semi-structured survey instrument. Int J Med Inform 2008;77(7):440-7.

35. Campbell EM, Guappone KP, Sittig DF, Dykstra RH, Ash JS. Computerized provider order entry adoption: implications for clinical workflow. J Gen Intern Med 2009;24(1):21-6.

36. Ash JS, Sittig DF, Dykstra R, Campbell E, Guappone K. The unintended consequences of computerized provider order entry: findings from a mixed methods exploration. Int J Med Inform 2009;78 Suppl 1:S69-76.

37. Pirnejad H, Bal R, Shahsavar N. The nature of unintended effects of health information systems concerning patient safety: a systematic review with thematic synthesis. Stud Health Technol Inform 2010;160(Pt 1):719-23.

38. Carling CL, Kirkehei I, Dalsbø TK, Paulsen E. Risks to patient safety associated with implementation of electronic applications for medication management in ambulatory care-a systematic review. BMC Med Inform Decis Mak 2013;13:133.

39. Voshall B, Piscotty R, Lawrence J, Targosz M. Barcode medication administration work-arounds: a systematic review and implications for nurse executives. J Nurs Adm 2013;43(10):530-5.

40. Gephart S, Carrington JM, Finley B. A systematic review of nurses' experiences with unintended consequences when using the electronic health record. Nurs Adm Q 2015;39(4):345-56.

41. Marcilly R, Ammenwerth E, Roehrer E, Pelayo S, Vasseur F, Beuscart-Zéphir MC. Usability flaws in medication alerting systems: impact on usage and work system. Yearb Med Inform 2015;10(1):55-67.

42. Kaushal R, Shojania KG, Bates DW. Effects of computerized physician order entry and clinical decision support systems on medication safety: a systematic review. Arch Intern Med 2003;163(12):1409-16

43. Chaudhry B, Wang J, Wu S, Maglione M, Mojica
W, Roth E, et al. Systematic review: impact of health information technology on quality, efficiency, and costs of medical care. Ann Intern Med 2006;144(10):742-52.

44. Shekelle PG, Morton SC, Keeler EB. Costs and benefits of health information technology. Evid Rep Technol Assess (Full Rep) 2006;(132):1-71.

45. Shebl NA, Franklin BD, Barber N. Clinical decision support systems and antibiotic use. Pharm World Sci 2007;29(4):342-9.

46. Eslami S, Abu-Hanna A, de Keizer NF. Evaluation of outpatient computerized physician medication order entry systems: a systematic review. J Am Med Inform Assoc 2007;14(4):400-6.

47. Eslami S, de Keizer NF, Abu-Hanna A. The impact of computerized physician medication order entry in hospitalized patients - a systematic review. Int J Med Inform 2008;77(6):365-76.

48. Wolfstadt JI, Gurwitz JH, Field TS, Lee M, Kalkar $\mathrm{S}, \mathrm{Wu} \mathrm{W}$, et al. The effect of computerized physician order entry with clinical decision support on the rates of adverse drug events: A systematic review. J Gen Intern Med 2008;23(4):451-8.

49. Ammenwerth E, Schnell-Inderst P, Machan C, Siebert U. The effect of electronic prescribing on medication errors and adverse drug events: a systematic review. J Am Med Inform Assoc 2008;15(5):585-600.

50. Yourman L, Concato J, Agostini JV. Use of computer decision support interventions to improve medication prescribing in older adults: a systematic review. Am J Geriatr Pharmacother 2008;6(2):119-29.

51. Bryan C, Boren SA. The use and effectiveness of electronic clinical decision support tools in the ambulatory/primary care setting: a systematic review of the literature. Inform Prim Care 2008;16(2):79-91.

52. Irani JS, Middleton JL, Marfatia R, Omana ET, D'Amico F. The use of electronic health records in the exam room and patient satisfaction: a systematic review. J Am Board Fam Med 2009;22(5):553-62.

53. Schedlbauer A, Prasad V, Mulvaney C, Phansalkar S, Stanton W, Bates DW, et al. What evidence supports the use of computerized alerts and prompts to improve clinicians' prescribing behavior? J Am Med Inform Assoc 2009;16(4):531-8.

54. Reckmann MH, Westbrook JI, Koh Y, Lo C, Day RO. Does computerized provider order entry reduce prescribing errors for hospital inpatients? A systematic review. J Am Med Inform Assoc 2009;16(5):613-23.

55. Mack EH, Wheeler DS, Embi PJ. Clinical decision support systems in the pediatric intensive care unit. Pediatr Crit Care Med 2009;10(1):23-8.

56. Bosman RJ. Impact of computerized information systems on workload in operating room and intensive care unit. Best Pract Res Clin Anaesthesiol 2009;23(1):15-26.

57. Mador RL, Shaw NT. The impact of a Critical Care Information System (CCIS) on time spent charting and in direct patient care by staff in the ICU: a review of the literature. Int J Med Inform 2009; 78(7):435-45.

58. van Rosse F, Maat B, Rademaker CM, van Vught AJ, Egberts AC, Bollen CW. The effect of computerized physician order entry on medication pre- 
A Survey of the Literature on Unintended Consequences Associated with Health Information Technology: 2014-2015

scription errors and clinical outcome in pediatric and intensive care: A systematic review. Pediatrics 2009;123(4):1184-90.

59. Pearson SA, Moxey A, Robertson J, Hains I, Williamson M, Reeve J, et al. Do computerised clinical decision support systems for prescribing change practice? A systematic review of the literature (1990-2007). BMC Health Serv Res 2009;9:154.

60. Vaziri A, Connor E, Shepherd I, Jones RT, Chan $\mathrm{T}$, de Lusignan S. Are we setting about improving the safety of computerised prescribing in the right way? A workshop report. Inform Prim Care 2009;17(3):175-82.

61. Hayward GL, Parnes AJ, Simon SR. Using health information technology to improve drug monitoring: a systematic review. Pharmacoepidemiol Drug Saf 2009;18(12):1232-7.

62. Goldzweig CL, Towfigh A, Maglione M, Shekelle PG. Costs and benefits of health information technology: new trends from the literature. Health Aff (Millwood) 2009;28(2):w282-93.

63. Niazkhani Z, Pirnejad H, Berg M, Aarts J. The impact of computerized provider order entry systems on inpatient clinical workflow: a literature review. J Am Med Inform Assoc 2009;16(4):539-49.

64. Weir CR, Staggers N, Phansalkar S. The state of the evidence for computerized provider order entry: a systematic review and analysis of the quality of the literature. Int J Med Inform 2009;78(6):365-74.

65. Jamal A, McKenzie K, Clark M. The impact of health information technology on the quality of medical and health care: a systematic review. HIM J 2009;38(3):26-37.

66. Berner ES. Clinical Decision Support Systems: State of the Art. AHRQ Publication No. 09-0069EF. Rockville, Maryland: Agency for Healthcare Research and Quality; June 2009.

67. Moxey A, Robertson J, Newby D, Hains I, Williamson M, Pearson SA. Computerized clinical decision support for prescribing: provision does not guarantee uptake. J Am Med Inform Assoc 2010;17(1):25-33.

68. Edwards A, Hollin I, Barry J, Kachnowski S. Barriers to cross-institutional health information exchange: a literature review. J Healthc Inf Manag 2010;24(3):22-34.

69. Minard JP, Turcotte SE, Lougheed MD. Asthma electronic medical records in primary care: an integrative review. J Asthma 2010;47(8):895-912.

70. Robertson J, Walkom E, Pearson SA, Hains I, Williamsone M, Newby D. The impact of pharmacy computerised clinical decision support on prescribing, clinical and patient outcomes: a systematic review of the literature. Int J Pharm Pract 2010;18(2):69-87.

71. Main C, Moxham T, Wyatt JC, Kay J, Anderson R, Stein K. Computerised decision support systems in order communication for diagnostic, screening or monitoring test ordering: systematic reviews of the effects and cost-effectiveness of systems. Health Technol Assess 2010;14(48):1-227.

72. Fontaine P, Ross SE, Zink T, Schilling LM. Systematic review of health information exchange in primary care practices. J Am Board Fam Med 2010;23(5):655-70.

73. Shojania KG, Jennings A, Mayhew A, Ramsay C, Eccles M, Grimshaw J. Effect of point-of-care computer reminders on physician behaviour: A systematic review. CMAJ 2010;182(5):E216-25.

74. Black AD, Car J, Pagliari C, Anandan C, Cresswell $\mathrm{K}$, Bokun T, et al. The impact of eHealth on the quality and safety of health care: a systematic overview. PLoS Med 2011;8(1):e1000387.

75. Sahota N, Lloyd R, Ramakrishna A, Mackay JA, Prorok JC, Weise-Kelly L, et al; CCDSS Systematic Review Team. Computerized clinical decision support systems for acute care management: a decision-maker-researcher partnership systematic review of effects on process of care and patient outcomes. Implement Sci 2011;6:91.

76. Holroyd-Leduc JM, Lorenzetti D, Straus SE, Sykes L, Quan H. The impact of the electronic medical record on structure, process, and outcomes within primary care: a systematic review of the evidence. J Am Med Inform Assoc 2011;18(6):732-7.

77. Roshanov PS, You JJ, Dhaliwal J, Koff D, Mackay JA, Weise-Kelly L, et al; CCDSS Systematic Review Team. Can computerized clinical decision support systems improve practitioners' diagnostic test ordering behavior? A decision-maker-researcher partnership systematic review. Implement Sci 2011;6:88

78. Handel DA, Wears RL, Nathanson LA, Pines JM. Using information technology to improve the quality and safety of emergency care. Acad Emerg Med 2011;18(6):e45-51.

79. McKibbon KA, Lokker C, Handler SM, Dolovich LR, Holbrook AM, O'Reilly D, et al. Enabling Medication Management Through Health Information Technology. Rockville (MD): Agency for Healthcare Research and Quality (US); 2011.

80. Tawadrous D, Shariff SZ, Haynes RB, Iansavichus AV, Jain AK, Garg AX. Use of clinical decision support systems for kidney-related drug prescribing: a systematic review. Am J Kidney Dis 2011;58(6):903-14.

81. Maslove DM, Rizk N, Lowe HJ. Computerized physician order entry in the critical care environment: a review of current literature. J Intensive Care Med 2011;26(3):165-71.

82. Nguyen L, Bellucci E, Nguyen LT. Electronic health records implementation: an evaluation of information system impact and contingency factors. Int J Med Inform 2014;83(11):779-96.

83. Lau F, Price M, Boyd J, Partridge C, Bell H, Raworth R. Impact of electronic medical record on physician practice in office settings: a systematic review. BMC Med Inform Decis Mak 2012;12:10.

84. Bright TJ, Wong A, Dhurjati R, Bristow E, Bastian L, Coeytaux RR, et al. Effect of clinical decision-support systems: a systematic review. Ann Intern Med 2012;157(1):29-43.

85. Cresswell K, Majeed A, Bates DW, Sheikh A. Computerised decision support systems for healthcare professionals: an interpretative review. Inform Prim Care 2012;20(2):115-28.

86. Kazmi Z. Effects of exam room EHR use on doctor-patient communication: a systematic literature review. Inform Prim Care 2013;21(1):30-9.

87. Baer HJ, Cho I, Walmer RA, Bain PA, Bates DW. Using electronic health records to address overweight and obesity: a systematic review. Am J Prev Med 2013;45(4):494-500.

88. Goldzweig CL, Orshansky G, Paige NM, Towfigh AA, Haggstrom DA, Miake-Lye I, et al. Electronic patient portals: evidence on health outcomes, satisfaction, efficiency, and attitudes: a systematic review. Ann Intern Med 2013;159(10):677-87.

89. Smith AJ, Skow Á, Bodurtha J, Kinra S. Health information technology in screening and treatment of child obesity: a systematic review. Pediatrics 2013;131(3):e894-902.

90. Georgiou A, Prgomet M, Paoloni R, Creswick N, Hordern A, Walter S, et al. The effect of computerized provider order entry systems on clinical care and work processes in emergency departments: a systematic review of the quantitative literature. Ann Emerg Med 2013;61(6):644-53.e16.

91. Liu J, Luo L, Zhang R, Huang T. Patient satisfaction with electronic medical/health record: a systematic review. Scand J Caring Sci 2013;27(4):785-91.

92. Lainer M, Mann E, Sönnichsen A. Information technology interventions to improve medication safety in primary care: a systematic review. Int $\mathrm{J}$ Qual Health Care 2013;25(5):590-8.

93. Nuckols TK, Smith-Spangler C, Morton SC, Asch SM, Patel VM, Anderson LJ, et al. The effectiveness of computerized order entry at reducing preventable adverse drug events and medication errors in hospital settings: a systematic review and meta-analysis. Syst Rev 2014;3:56.

94. Boyle R, Solberg L, Fiore M. Use of electronic health records to support smoking cessation. Cochrane Database Syst Rev 2014;12:CD008743.

95. Rudin RS, Motala A, Goldzweig CL, Shekelle PG. Usage and effect of health information exchange: a systematic review. Ann Intern Med 2014;161(11):803-11.

96. Fasola G, Macerelli M, Follador A, Rihawi K, Aprile G, Della Mea V. Health information technology in oncology practice: a literature review. Cancer Inform 2014;13:131-9.

97. Ranji SR, Rennke S, Wachter RM. Computerised provider order entry combined with clinical decision support systems to improve medication safety: a narrative review. BMJ Qual Saf 2014;23(9):773-80.

98. Rahurkar S, Vest JR, Menachemi N. Despite the spread of health information exchange, there is little evidence of its impact on cost, use, and quality of care. Health Aff (Millwood) 2015;34(3):477-83.

99. Vimalananda VG, Gupte G, Seraj SM, Orlander J, Berlowitz D, Fincke BG, et al. Electronic consultations (e-consults) to improve access to specialty care: a systematic review and narrative synthesis. J Telemed Telecare 2015;21(6):323-30.

100. Rubbo B, Fitzpatrick NK, Denaxas S, Daskalopoulou M, Yu N, Patel RS; UK Biobank Follow-up and Outcomes Working Group, Hemingway $\mathrm{H}$. Use of electronic health records to ascertain, validate and phenotype acute myocardial infarction: a systematic review and recommendations. Int J Cardiol 2015;187:705-11.

101. Campanella P, Lovato E, Marone C, Fallacara L, Mancuso A, Ricciardi W, et al. The impact of electronic health records on healthcare quality: a systematic review and meta-analysis. Eur J Public Health 2015;pii:ckv122.

102. Buntin MB, Burke MF, Hoaglin MC, Blumenthal D. The benefits of health information technology: a review of the recent literature shows predominantly positive results. Health Aff (Millwood) 2011;30(3):464-71.

103. Georgiou A, Prgomet M, Markewycz A, Adams 
E, Westbrook JI. The impact of computerized provider order entry systems on medical-imaging services: a systematic review. J Am Med Inform Assoc 2011;18(3):335-40.

104. Shekelle P.G., Jones SS, Rudin BS, Shanman R, Timmer M, Perry TR, et al. Health Information Technology: An Updated Systematic Review with a Focus on Meaningful Use Functionalities. (Prepared by the Southern California-RAND Evidence-based Practice Center under Contract No. HHSP23337020T). Office of the National Coordinator for Health Information Technology. Washington, D.C.; 2014

105. Jones SS, Rudin RS, Perry T, Shekelle PG. Health information technology: an updated systematic review with a focus on meaningful use. Ann Intern Med 2014;160(1):48-54.

106. Kellermann AL, Jones SS. What it will take to achieve the as-yet-unfulfilled promises of health information technology. Health Aff (Millwood) 2013;32(1):63-8.

107. Schildcrout JS, Shi Y, Danciu I, Bowton E, Field JR, Pulley JM, et al. A prognostic model based on readily available clinical data enriched a pre-emptive pharmacogenetic testing program. J Clin Epidemiol 2016 Apr;72:107-15.

108. Hanauer DA, Mei Q, Law J, Khanna R, Zheng K. Supporting information retrieval from electronic health records: a report of University of Michigan's nine-year experience in developing and using the Electronic Medical Record Search Engine (EMERSE). J Biomed Inform 2015;55:290-300.

109. Mehrabi S, Krishnan A, Sohn S, Roch AM, Schmidt H, Kesterson J, Beesley et al. DEEPEN: A negation detection system for clinical text incorporating dependency relation into NegEx. J Biomed Inform 2015;54:213-9.

110. Lohmann K, Gartner D, Kurze R, Schösler T, Schwald M, Störzinger D, et al. More than just crushing: a prospective pre-post intervention study to reduce drug preparation errors in patients with feeding tubes. J Clin Pharm Ther 2015;40(2):220-5.

111. Ryan AM, McCullough CM, Shih SC, Wang JJ, Ryan MS, Casalino LP. The intended and unintended consequences of quality improvement interventions for small practices in a community-based electronic health record implementation project. Med Care 2014;52(9):826-32.

112. Amland RC, Dean BB, Yu H, Ryan H, Orsund T, Hackman JL, et al. computerized clinical decision support to prevent venous thromboembolism among hospitalized patients: proximal outcomes from a multiyear quality improvement project. J Healthc Qual 2015;37(4):221-31.

113. Sockolow PS, Rogers M, Bowles KH, Hand KE, George J. Challenges and facilitators to nurse use of a guideline-based nursing information system: recommendations for nurse executives. Appl Nurs Res 2014;27(1):25-32.

114. Pell JM, Cheung D, Jones MA, Cumbler E. Don't fuel the fire: decreasing intravenous haloperidol use in high risk patients via a customized electronic alert. J Am Med Inform Assoc 2014;21(6):1109-12.

115. Russell RA, Triscari D, Murkowski K, Scanlon MC. Impact of computerized order entry to pharmacy interface on order-infusion pump discrepancies. J Drug Deliv 2015;2015:686598.

116. Rizzato Lede DA, Benítez SE, Mayan JC 3rd, Smith MI, Baum AJ, Luna DR, et al. Patient safety at transitions of care: use of a compulsory electronic reconciliation tool in an academic hospital. Stud Health Technol Inform 2015;216:232-6.

117. Overhage JM, Gandhi TK, Hope C, Seger AC, Murray MD, Orav EJ, et al. Ambulatory computerized prescribing and preventable adverse drug events. J Patient Saf 2016 Jun;12(2):69-74.

118. Cresswell KM, Bates DW, Williams R, Morrison Z, Slee A, Coleman J, et al. Evaluation of medium-term consequences of implementing commercial computerized physician order entry and clinical decision support prescribing systems in two 'early adopter' hospitals. J Am Med Inform Assoc 2014;21(e2):e194-202.

119. Nanji KC, Rothschild JM, Boehne JJ, Keohane CA, Ash JS, Poon EG. Unrealized potential and residual consequences of electronic prescribing on pharmacy workflow in the outpatient pharmacy. J Am Med Inform Assoc 2014;21(3):481-6.

120. Graber ML, Siegal D, Riah H, Johnston D, Kenyon K. Electronic health record-related events in medical malpractice claims. J Patient Saf 2015. [Epub ahead of print]

121. Magrabi F, Baker M, Sinha I, Ong MS, Harrison S, Kidd MR, et al. Clinical safety of England's national programme for IT: a retrospective analysis of all reported safety events 2005 to 2011 . Int J Med Inform 2015;84(3):198-206.

122. Meeks DW, Smith MW, Taylor L, Sittig DF, Scott JM, Singh H. An analysis of electronic health record-related patient safety concerns. J Am Med Inform Assoc 2014;21(6):1053-9.

123. Risko N, Anderson D, Golden B, Wasil E, Barrueto F, Pimentel L, et al. The impact of electronic health record implementation on emergency physician efficiency and patient throughput. Healthc (Amst) 2014;2(3):201-4.

124. Ward MJ, Froehle CM, Hart KW, Collins SP, Lindsell CJ. Transient and sustained changes in operational performance, patient evaluation, and medication administration during electronic health record implementation in the emergency department. Ann Emerg Med 2014;63(3):320-8.

125. Benda NC, Meadors ML, Zachary Hettinger A, Ratwani RM. Emergency physician task switching increases with the introduction of a commercial electronic health record. Ann Emerg Med 2016 Jun;67(6):741-6.

126. Tall JM, Hurd M, Gifford T. Minimal impact of an electronic medical records system. Am J Emerg Med 2015;33(5):663-6.

127. MacMillan TE, Slessarev M, Etchells E. eWasted time: Redundant work during hospital admission and discharge. Health Informatics J 2016;22(1):60-6

128. Redd TK, Read-Brown S, Choi D, Yackel TR, Tu DC, Chiang MF. Electronic health record impact on productivity and efficiency in an academic pediatric ophthalmology practice. J AAPOS 2014;18(6):584-9.

129. Sanders DS, Read-Brown S, Tu DC, Lambert WE, Choi D, Almario BM, et al. Impact of an electronic health record operating room management system in ophthalmology on documentation time, surgical volume, and staffing. JAMA
Ophthalmol 2014;132(5):586-92

130. Georgiou A, Prgomet M, Lymer S, Hordern A, Ridley L, Westbrook J. The Impact of a Health IT changeover on medical imaging department work processes and turnaround times: a mixed method study. Appl Clin Inform 2015;6(3):443-53.

131. Carayon P, Wetterneck TB, Alyousef B, Brown RL, Cartmill RS, McGuire K, et al. Impact of electronic health record technology on the work and workflow of physicians in the intensive care unit. Int J Med Inform 2015;84(8):578-94.

132. Victores AJ, Coggins K, Takashima M. Electronic health records and resident workflow: a time-motion study of otolaryngology residents. Laryngoscope 2015;125(3):594-8.

133. Cifuentes M, Davis M, Fernald D, Gunn R, Dickinson P, Cohen DJ. Electronic health record challenges, workarounds, and solutions observed in practices integrating behavioral health and primary care. J Am Board Fam Med 2015;28 Suppl 1:S63-72.

134. Lafata JE, Shay LA, Brown R, Street RL. Office-based tools and primary care visit communication, length, and preventive service delivery. Health Serv Res 2016 Apr;51(2):728-45

135. McLean TA, Lewkowitz AK, Test E, Zlatnik MG. Does an electronic health record improve completeness of prenatal studies? Appl Clin Inform 2015;6(4):669-76.

136. Thirukumaran CP, Dolan JG, Reagan Webster P, Panzer RJ, Friedman B. The impact of electronic health record implementation and use on performance of the Surgical Care Improvement Project measures. Health Serv Res 2015;50(1):273-89.

137. Varpio L, Day K, Elliot-Miller P, King JW, Kuziemsky C, Parush A, et al. The impact of adopting EHRs: how losing connectivity affects clinical reasoning. Med Educ 2015;49(5):476-86.

138. Melby L, Hellesø R. Introducing electronic messaging in Norwegian healthcare: unintended consequences for interprofessional collaboration. Int J Med Inform 2014;83(5):343-53.

139. Saddik B, Al-Mansour S. Does CPOE support nurse-physician communication in the medication order process? A nursing perspective. Stud Health Technol Inform 2014;204:149-55.

140. Ser G, Robertson A, Sheikh A. A qualitative exploration of workarounds related to the implementation of national electronic health records in early adopter mental health hospitals. PLoS One 2014(1):e77669.

141. Fleming NS, Becker ER, Culler SD, Cheng D, McCorkle R, da Graca B, et al. The impact of electronic health records on workflow and financial measures in primary care practices. Health Serv Res 2014:49(1 Pt 2):405-20.

142. Howley MJ, Chou EY, Hansen N, Dalrymple PW. The long-term financial impact of electronic health record implementation. J Am Med Inform Assoc 2015;22(2):443-52.

143. Hysong SJ, Spitzmuller C, Espadas D, Sittig DF, Singh $\mathrm{H}$. Electronic alerts and clinician turnover: the influence of user acceptance. Am J Manag Care 2014;20(11 Spec No. 17):SP520-30.

144. Crowson MG, Vail C, Eapen RJ. Influence of electronic medical record implementation on provider retirement at a major academic medical centre. J Eval Clin Pract 2016 Apr;22(2):222-6. 
145. Campos-Castillo C, Anthony DL. The double-edged sword of electronic health records: implications for patient disclosure. J Am Med Inform Assoc 2015;22(e1):e130-40.

146. Carrington JM, Gephart SM, Verran JA, Finley BA. Development of an instrument to measure the unintended consequences of EHRs. West J Nurs Res 2015;37(7):842-58.

147. Patel VL, Arocha JF, Kushniruk AW. Patients' and physicians' understanding of health and biomedical concepts: relationship to the design of EMR systems. J Biomed Inform 2002;35(1):8-16.

148. Canon SJ, Purifoy JA, Heulitt GM, Hogan W, Swearingen C, Williams M, et al. Results: Survey of pediatric urology electronic medical records-use and perspectives. J Urol 2011;186(4 Suppl):1740-4.

149. Novak LL, Anders S, Gadd CS, Lorenzi NM. Mediation of adoption and use: a key strategy for mitigating unintended consequences of health IT implementation. J Am Med Inform Assoc 2012;19(6):1043-9.

150. Health Information Technology Patient Safety Action \& Surveillance Plan. Washington, DC: Office of the National Coordinator for Health Information Technology. 2013.

151. Sittig DF, Singh H. Electronic health records and national patient-safety goals. N Engl J Med 2012;367(19):1854-60.

152. Magrabi F, Aarts J, Nohr C, Baker M, Harrison S, Pelayo S, Talmon et al. A comparative review of patient safety initiatives for national health information technology. Int J Med Inform 2013;82(5):e139-48.

153. Wallace C, Zimmer KP, Possanza L, Giannini R, Solomon R. How to Identify and Address Unsafe Conditions Associated with Health IT. (Prepared by ECRI Institute under Contract No. HHSP23320095655WC, Task Order HHSP23337003T). Washington, DC: Office of the National Coordinator for Health Information Technology; 2013.

154. Schneider EC, Ridgely MS, Meeker D, Hunter
LE, Khodyakov D, Rudin R. Promoting Patient Safety Through Effective Health Information Technology Risk Management. (Prepared by RAND Corporation under Contract No. HHSP23320095649WC). Washington, DC: Office of the National Coordinator for Health Information Technology; 2014.

155. Ratwani R, Hettinger AZ, Fairbanks RJ. The Role of Health IT Developers in Improving Patient Safety in High Reliability Organizations. (Prepared by National Center for Human Factors in Healthcare under Contract No. HHSP23320095655WC, Task Order HHSP23337003T). Washington, DC: Office of the National Coordinator for Health Information Technology; 2014.

156. Mardon R, Olinger L, Szekendi M, Williams T, Sparnon E, Zimmer K. Health Information Technology Adverse Event Reporting: Analysis of Two Databases. (Prepared by Westat, UHC, and ECRI Institute under Contract Order No. HHSP23337024T). Washington, DC: Office of the National Coordinator for Health Information Technology; 2014.

157. Castro G, Buczkowski L, Hafner J, Barrett S, Rasinski K, Williams S. Investigations of HealthIT-Related Deaths, Serious Injuries, or Unsafe Conditions. (Prepared by Joint Commission under Contract No. HHSP233201300019C). Washington, DC: Office of the National Coordinator for Health Information Technology; 2015

158. SAFER Guides. https://www.healthit.gov/safer/ safer-guides; accessed February 1, 2015.

159. Lowry SZ, Quinn MT, Ramaiah M, Schumacher RM, Patterson ES, North R, et al. Technical Evaluation, Testing, and Validation of the Usability of Electronic Health Records. NISTIR 7804. Washington DC: National Institute of Standards and Technology; 2012.

160. Lowry SZ, Ramaiah M, Taylor S, Patterson ES, Prettyman SS, Simmons D, et al. Technical Evaluation, Testing, and Validation of the Usability of Electronic Health Records: Empirically
Based Use Cases for Validating Safety Enhanced Usability and Guidelines for Standardization. NISTIR 7804-1. Washington DC: National Institute of Standards and Technology; 2015.

161. U.S. Food and Drug Administration. FDASIA Health IT Report Proposed Strategy and Recommendations for a Risk-Based Framework; 2014.

162. National Quality Forum. Identification and Prioritization of Health IT Patient Safety Measures. (Prepared under Contract No. HHSM-500-201200009I, Task Order HHSM-500-T0016). Washington DC: Department of Health and Human Services; 2016

163. Health IT Safety Collaborative. http://www. healthitsafety.org/; accessed February 1, 2015.

164. The Partnership for Health IT Patient Safety. https://www.ecri.org/resource-center/Pages/ HITPartnership.aspx; accessed February 1, 2015.

165. CHIME National Patient ID Challenge. https:// herox.com/PatientIDChallenge; accessed February $1,2015$.

166. Rosenbaum L. Transitional chaos or enduring harm? The EHR and the disruption of medicine. N Engl J Med 2015;373(17):1585-8.

167. Sittig DF, Classen DC, Singh H. Patient safety goals for the proposed Federal Health Information Technology Safety Center. J Am Med Inform Assoc 2015;22(2):472-8.

168. Singh H, Sittig DF. Measuring and improving patient safety through health information technology: The Health IT Safety Framework. BMJ Qual Saf 2016 Apr;25(4):226-32.

169. Wachter R. The Digital Doctor: Hope, Hype, and Harm at the Dawn of Medicine's Computer Age. New York: McGraw-Hill; 2015.

\section{Correspondence to:}

Kai Zheng PhD

5228 Donald Bren Hall

Irvine, CA 92697-3440

USA

E-mail: kai.zheng@uci.edu 